\title{
Water and ammonia abundances in S140 with the Odin ${ }^{\star}$ satellite ${ }^{\star \star}$
}

\author{
C. M. Persson ${ }^{1}$, M. Olberg ${ }^{1}$, Å. Hjalmarson ${ }^{1}$, M. Spaans ${ }^{2}$, J. H. Black ${ }^{1}$, U. Frisk ${ }^{3}$, T. Liljeström ${ }^{4}$, \\ A. O. H. Olofsson ${ }^{1,5}$, D. R. Poelman ${ }^{6}$, and Aa. Sandqvist ${ }^{7}$ \\ 1 Onsala Space Observatory, Chalmers University of Technology, 43992 Onsala, Sweden \\ e-mail: carina.persson@chalmers.se \\ 2 Kapteyn Astronomical Institute, Rijksuniversiteit Groningen, PO Box 800, 9700 AV Groningen, The Netherlands \\ 3 Swedish Space Corporation, PO Box 4207, 17104 Solna, Sweden \\ 4 Metsähovi Radio Observatory, Helsinki University of Technology, Otakaari 5A, 02150 Espoo, Finland \\ 5 GEPI, Observatoire de Paris, CNRS, 5 place Jules Janssen, 92195 Meudon, France \\ 6 SUPA, School of Physics and Astronomy, University of St Andrews, North Haugh, St Andrews KY16 9SS, UK \\ 7 Stockholm Observatory, AlbaNova University Center, 10691 Stockholm, Sweden
}

Received 7 September 2008 / Accepted 14 November 2008

ABSTRACT

\begin{abstract}
Aims. We investigate the effect of the physical environment on water and ammonia abundances across the S140 photodissociation region (PDR) with an embedded outflow.

Methods. We used the Odin satellite to obtain strip maps of the ground-state rotational transitions of ortho-water and ortho-ammonia, as well as $\mathrm{CO}(5-4)$ and ${ }^{13} \mathrm{CO}(5-4)$ across the $\mathrm{PDR}$, and $\mathrm{H}_{2}^{18} \mathrm{O}$ in the central position. A physi-chemical inhomogeneous PDR model was used to compute the temperature and abundance distributions for water, ammonia, and $\mathrm{CO}$. A multi-zone escape probability method then calculated the level populations and intensity distributions. These results are compared to a homogeneous model computed with an enhanced version of the RADEX code.

Results. $\mathrm{H}_{2} \mathrm{O}, \mathrm{NH}_{3}$, and ${ }^{13} \mathrm{CO}$ show emission from an extended PDR with a narrow line width of $\sim 3 \mathrm{~km} \mathrm{~s}^{-1}$. Like $\mathrm{CO}$, the water line profile is dominated by outflow emission, but mainly in the red wing. Even though CO shows strong self-absorption, no signs of self-absorption are seen in the water line. The $\mathrm{H}_{2}^{18} \mathrm{O}$ molecule is not detected. The PDR model suggests that the water emission arises mainly from the surfaces of optically thick, high-density clumps with $n\left(\mathrm{H}_{2}\right) \gtrsim 10^{6} \mathrm{~cm}^{-3}$ and a clump water abundance, with respect to $\mathrm{H}_{2}$, of $5 \times 10^{-8}$. The mean water abundance in the PDR is $5 \times 10^{-9}$ and between $\sim 4 \times 10^{-8}-4 \times 10^{-7}$ in the outflow derived from a simple two-level approximation. The RADEX model points to a somewhat higher average PDR water abundance of $1 \times 10^{-8}$. At low temperatures deep in the cloud, the water emission is weaker, likely due to adsorption onto dust grains, while ammonia is still abundant. Ammonia is also observed in the extended clumpy PDR, likely from the same high density and warm clumps as water. The average ammonia abundance is about the same as for water: $4 \times 10^{-9}$ and $8 \times 10^{-9}$ given by the PDR model and RADEX, respectively. The differences between the models most likely arise from uncertainties in density, beam-filling, and volume-filling of clumps. The similarity of water and ammonia PDR emission is also seen in the almost identical line profiles observed close to the bright rim. Around the central position, ammonia also shows some outflow emission, although weaker than water in the red wing. Predictions of the $\mathrm{H}_{2} \mathrm{O} 1_{1,0}-1_{0,1}$ and $1_{1,1}-0_{0,0}$ antenna temperatures across the PDR are estimated with our PDR model for the forthcoming observations with the Herschel Space Observatory.
\end{abstract}

Key words. ISM: abundances - ISM: individual objects: S140 - ISM: molecules - submillimeter - line: profiles - line: formation

\section{Introduction}

The chemistry and the evolution of the interstellar medium depend to a high degree on the available amount of carbon and oxygen. The carbon-based chemistry is understood fairly well, but the oxygen chemistry is not. Two molecules vital to understanding the oxygen chemistry are water and molecular oxygen, since some chemical models predict that a large fraction of oxygen could be in the form of these molecules.

Observations of the ground-state rotational water transition with NASA's Submillimeter Wave Astronomy Satellite (SWAS; Melnick et al. 2000b) and the Odin satellite (Nordh et al. 2003)

\footnotetext{
* Odin is a Swedish-led satellite project funded jointly by the Swedish National Space Board (SNSB), the Canadian Space Agency (CSA), the National Technology Agency of Finland (Tekes) and Centre National d'Etudes Spatiales (CNES). The Swedish Space Corporation was the prime contractor and also is responsible for the satellite operation.

$\star \star$ Appendices A and B are available in electronic form at

http://www . aanda. org
}

have both shown very low abundances of molecular oxygen, $5 \times 10^{-8}$ (Larsson et al. 2007), and water, $10^{-8}-10^{-9}$ (Olofsson et al. 2003), in the extended cold clouds in contrast to the predictions from chemical models. However, on smaller scales in warm or shocked regions and outflows, where the density is higher than $10^{5}-10^{6} \mathrm{~cm}^{-3}$, SWAS (e.g. Melnick et al. 2000a) and Odin (e.g. Persson et al. 2007) have also shown locally increased water abundance by several orders of magnitude, $10^{-6}-10^{-4}$ with respect to molecular hydrogen. The analysis of the data is, however, somewhat difficult and uncertain due to observations of a single line and large beam-widths, but is in agreement with observations of a wealth of highly excited water vapour transitions with ISO (the Infrared Space Observatory, Cernicharo \& Crovisier 2005). These locally high abundances support the predictions that water should be an important cooling agent in the star-forming process, especially at high temperatures and densities (e.g. Neufeld et al. 1995; Bergin et al. 2003). The strong dependence of the water chemistry on the temperature changes seen in star-forming regions also make water an excellent tracer 
of shocks and outflows from protostars still hidden within their dusty envelopes (e.g. Bergin et al. 1998).

The intention of this paper is to continue the previous work with ISO, SWAS, and Odin, and also to be a valuable input to future observations with the Heterodyne Instrument for the Far Infrared (HIFI) on board the Herschel ${ }^{1}$ satellite to be launched in 2009. Our aim is to investigate how the water abundance depends on the environment in a star-forming region. The high spectral resolution $\left(0.5 \mathrm{~km} \mathrm{~s}^{-1}\right)$ and the smaller beam-size of Odin compared to SWAS $(2.1$ vs. $3.3 \times 4.5)$, which enable higher spatial resolution maps, allow a more detailed analysis of the line profile observed at each map position. We can also obtain an estimate of the beam-filling by comparing SWAS and Odin line profiles if the same object has been observed. If not properly taken into account the beam-filling may otherwise influence the results by orders of magnitude.

In addition to the water observations a second goal of our work is to perform and analyse observations of the important ground-state rotational transition of ammonia. While ammonia has been extensively observed in the inversion transitions at cm-wavelengths (e.g. Ungerechts et al. 1986; Zhou et al. 1993), the $1_{0}-0_{0}$ transition, however, cannot be observed from ground due to the opaque terrestrial atmosphere. The first observation of this transition was performed by the Kuiper Airborne Observatory (Keene et al. 1983), and was later followed up with Odin (Larsson et al. 2003; Liseau et al. 2003; Olofsson et al. 2007).

Our chosen target is the well known molecular cloud and bright nebula Sh 2-140, more commonly known as S140 (Sharpless 1959; Blair et al. 1978). It is located at a distance of 910 pc (Crampton \& Fisher 1974) in the large L1202/L1204 dark cloud. This region is illuminated from the south-western side by the nearby B0.5V star HD 211880, creating a bright $\mathrm{HII}$ region and an almost edge-on PDR adjacent to the molecular cloud and extending deeply into the cloud. The core in S140 also contains a small cluster of deeply embedded young stellar objects which illuminate the cloud from within. One or more of these early B-stars are undergoing some mass-loss process and molecular outflows are observed from this dense and warm region (e.g. Rouan et al. 1977; Hayashi et al. 1987; Minchin et al. 1993; Preibisch \& Smith 2002). The brightest infrared source in S140, called IRS1, is on small scales composed of several sources, two of which might be powering bipolar molecular outflows in almost perpendicular directions (Trinidad et al. 2007). The increased temperature and density in both the PDR and the outflows are likely expected to produce a higher than average water abundance. For these reasons, S140 is a promising target for our purposes.

Both ISO (Boonman \& van Dishoeck 2003) and SWAS (Snell et al. 2000a; Ashby et al. 2000a,b; Franklin et al. 2008) have observed water in S140 and several attempts have been made to model these observations (e.g. Ashby et al. 2000a,b; Boonman et al. 2003; Poelman \& Spaans 2005, 2006). However, they all have had difficulties to explain the unusual, narrow $1_{1,0}-1_{0,1}$ emission line with no or little evidence of selfabsorption. In order to obtain more information about the water emission we have conducted strip map observations of S140, starting deep within the molecular cloud and reaching outside the bright rim (see Fig. 1). Strip maps are also simultaneously performed of the previously unobserved $\mathrm{NH}_{3}\left(1_{0}-0_{0}\right)$ transition.

To aid the analysis of water and ammonia, we have in addition performed $\mathrm{H}_{2}^{18} \mathrm{O}$ observations in the central position, strip

1 http://herschel.esac.esa.int/

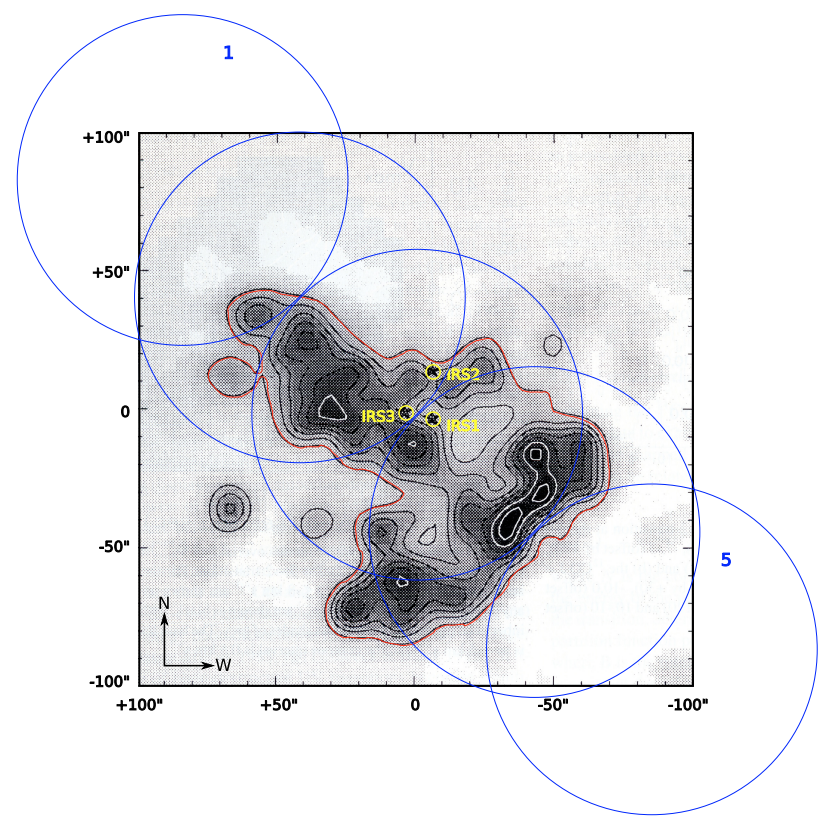

Fig. 1. A CI map (492 GHz) of S140 observed with JCMT (Minchin et al. 1993). The Odin 2'.1 beam at the five strip positions is also shown. Position 1 in NE to position 5 in SW.

maps of $\mathrm{CO}(5-4)$ and ${ }^{13} \mathrm{CO}(5-4)$ with the Odin satellite, and a ${ }^{13} \mathrm{CO}(1-0)$ map with the Onsala $20-\mathrm{m}$ telescope.

\section{Observations}

All transitions, except ${ }^{13} \mathrm{CO}(1-0)$, were acquired with the Odin satellite from 2003 to 2006 with a total of 561 orbits (see Table 1). The calibration procedure (the chopper wheel method) is described in Olberg et al. (2003). The Odin $1.1 \mathrm{~m}$ offset Gregorian telescope has a circular beam at $557 \mathrm{GHz}$ with a Full Width of Half Maximum (FWHM) of 2'.1 (Frisk et al. 2003). Being outside the atmosphere, and with an exceptionally high main beam efficiency, $\eta_{\mathrm{mb}}=0.9$, our intensity calibration is very accurate. The intensity scale in the figures is expressed in terms of antenna temperature $T_{\mathrm{A}}^{*}$. In all calculations, however, the main beam efficiency is properly taken into account. The reconstructed pointing offset was $<15^{\prime \prime}$ during most of the time. The simultaneous observations of $\mathrm{H}_{2} \mathrm{O}$ and $\mathrm{NH}_{3}$, and of ${ }^{13} \mathrm{CO}$, $\mathrm{CO}$ and $\mathrm{H}_{2} \mathrm{O}$ guarantee their pointing to be identical.

Three different tunable submm receivers were used, having average single-sideband (SSB) system temperatures of about $3300 \mathrm{~K}$. They were used in combination with a hybrid autocorrelator spectrometer (300-400 MHz working bandwidth) and an acousto-optical spectrometer ( $1 \mathrm{GHz}$ working bandwidth), both with a channel spacing of $\sim 0.3 \mathrm{~km} \mathrm{~s}^{-1}$. All observations were performed in the sky-switching mode with reference sky beams at $42^{\circ}$ distance and $4.4^{\circ} \mathrm{FWHM}$. To correct for ripple, observations of a reference off position 30' east of S140 were conducted as well.

The five point strip maps have $60^{\prime \prime}$ step size with strip centre coordinates RA 22. $17.42^{\mathrm{s}} .0$, Dec +63.03.45'.0 (B1950) at the cloud core (our position 3, Fig. 1). Position 1 is in NE and position 5 in SW. The frequency scale is set relative to a source LSR velocity of $-7.5 \mathrm{~km} \mathrm{~s}^{-1}$.

In addition, the Onsala 20-m telescope was used in 2006 for a ${ }^{13} \mathrm{CO}(1-0) 10 \times 11$ point map of the S140 molecular cloud with a spacing of $30^{\prime \prime}$. The SSB system temperature was $\sim 500 \mathrm{~K}$, the 
Table 1. All molecular transitions are observed with the Odin satellite except the ${ }^{13} \mathrm{CO}(1-0)$ map which is observed with the Onsala $20-\mathrm{m}$ telescope.

\begin{tabular}{llrrlr}
\hline \hline Species & Transition & $\begin{array}{r}\text { Freq. } \\
{[\mathrm{MHz}]}\end{array}$ & $\begin{array}{r}E_{\mathrm{u}}^{a} \\
{[\mathrm{~K}]}\end{array}$ & $\begin{array}{l}n_{\text {crit }}^{b} \\
{\left[\mathrm{~cm}^{-3}\right]}\end{array}$ & $\begin{array}{r}\text { Int. time } \\
{[\mathrm{h}]}\end{array}$ \\
\hline $\mathrm{H}_{2} \mathrm{O}$ & $1_{1,0}-1_{0,1}$ & 556936.0 & 61 & $3 \times 10^{8 c}$ & 41 \\
$\mathrm{H}_{2}^{18} \mathrm{O}$ & $1_{1,0}-1_{0,1}$ & 547676.4 & 60 & $3 \times 10^{8 c}$ & 91 \\
$\mathrm{CO}$ & $5-4$ & 576267.9 & 83 & $2 \times 10^{5 c}$ & 9 \\
${ }^{13} \mathrm{CO}$ & $5-4$ & 550926.3 & 79 & $2 \times 10^{5}$ & 9 \\
${ }^{13} \mathrm{CO}$ & $1-0$ & 110210.4 & 5 & $7 \times 10^{2}$ & $2 \mathrm{~min} /$ pos \\
$\mathrm{NH}_{3}$ & $1_{0}-0_{0}$ & 572498.1 & 29 & $5 \times 10^{7 c}$ & 16 \\
\hline
\end{tabular}

${ }^{a}$ Upper state energy; ${ }^{b}$ critical density calculated for a temperature of $40 \mathrm{~K} ;{ }^{c}$ can be considerably reduced because of radiative trapping.

main-beam efficiency 0.5 , and the FWHM beam at $110.201 \mathrm{GHz}$ is $34^{\prime \prime}$.

\section{Results}

The observed Odin spectra of $\mathrm{CO}, \mathrm{H}_{2} \mathrm{O}$, and $\mathrm{NH}_{3}$ are shown together with ${ }^{13} \mathrm{CO}(5-4)$ in Figs. 2-4 to allow a comparison of the line profiles of the different molecules at each position. The water and ammonia spectra are also shown together in Fig. 5. No $\mathrm{H}_{2}^{18} \mathrm{O}$ emission was detected in the central position (Fig. B.1, online material) at an rms noise level of $8 \mathrm{mK}$. Tables with $1 \sigma \mathrm{rms}$, integrated intensities, peak antenna temperatures, line widths and amplitudes of Gaussian fits, and centre velocities for species observed with Odin are found in the on-line Tables B.1-B.4. The on-line material also includes figures of Gaussian fits to ${ }^{13} \mathrm{CO}(1-0)$ and $J=5-4, \mathrm{H}_{2} \mathrm{O}$ and $\mathrm{NH}_{3}\left(1_{1}-0_{0}\right)$ (Figs. B.4-B.7).

Below we will discuss the observations and some straight forward results, while the model results for the narrow PDR component are presented in Sects. 4 and 5.

\subsection{Carbon monoxide}

The $\mathrm{CO}(5-4)$ and ${ }^{13} \mathrm{CO}(5-4)$ observations show a narrow line from the PDR in all positions superimposed on a broader outflow feature seen in $\mathrm{CO}$ at positions $1-4$ and in ${ }^{13} \mathrm{CO}$ at the centre position (Fig. 2). The emission peaks about $20^{\prime \prime}$ from the central position towards position 2 deeper in the cloud. A comparison of the ${ }^{12} \mathrm{CO}$ and ${ }^{13} \mathrm{CO}$ line shapes suggests strong $\mathrm{CO}(5-4)$ selfabsorption by lower excitation foreground gas. This is also discussed by e.g. Minchin et al. (1993) and Boonman et al. (2003) where the higher $J$-level CO lines are strongly self-absorbed. No strong self-absorption is visible in ${ }^{13} \mathrm{CO}$.

The mean opacity of ${ }^{12} \mathrm{CO}$ and ${ }^{13} \mathrm{CO}$ can be determined by comparison of their antenna peak temperatures if the isotopologue abundance ratio $R$ is known. Assuming that the opacity of ${ }^{12} \mathrm{CO}$ is larger than the opacity of ${ }^{13} \mathrm{CO}$ by a factor $R$, we will have

$\frac{T_{\mathrm{A}, 13}^{*}}{T_{\mathrm{A}, 12}^{*}}=\frac{J\left(T_{\mathrm{ex}}^{13}\right)\left(1-\mathrm{e}^{-\tau_{13}}\right) \eta_{\mathrm{bf}, 13}}{J\left(T_{\mathrm{ex}}^{12}\right)\left(1-\mathrm{e}^{-\tau_{12}}\right) \eta_{\mathrm{bf}, 12}}=\frac{J\left(T_{\mathrm{ex}}^{13}\right)\left(1-\mathrm{e}^{-\tau_{13}}\right) \eta_{\mathrm{bf}, 13}}{J\left(T_{\mathrm{ex}}^{12}\right)\left(1-\mathrm{e}^{-R \tau_{13}}\right) \eta_{\mathrm{bf}, 12}},(1)$

where the radiation temperature, $J\left(T_{\mathrm{ex}}\right)$, is a function of the excitation temperature, $T_{\mathrm{ex}}$,

$J\left(T_{\mathrm{ex}}\right)=\frac{h v}{k} \frac{1}{\mathrm{e}^{h v / k T_{\text {ex }}}-1}$.
The beam-filling factor is calculated with

$\eta_{\mathrm{bf}}=\frac{\theta_{\mathrm{s}}^{2}}{\theta_{\mathrm{s}}^{2}+\theta_{\mathrm{mb}}^{2}}$

assuming that both the source brightness distribution and the antenna response are circularly symmetric and Gaussian.

Assuming that the excitation temperatures are the same for both species, co-spatial emissions and using $R=70$, the opacities of the narrow component of ${ }^{12} \mathrm{CO}$ and ${ }^{13} \mathrm{CO}$ are estimated to be $\$ 70$ and $\lesssim 1$, while the opacities in the broad outflow component are $\lesssim 7$ and $\lesssim 0.1$, respectively. Due to the self-absorption of ${ }^{12} \mathrm{CO}$ the opacities are upper limits.

The size of the emission region can be estimated by comparing Odin and SWAS antenna temperatures

$\frac{T_{\mathrm{A}, \text { Odin }}^{*}}{T_{\mathrm{A}, \mathrm{SWAS}}^{*}}=\frac{(3.3 \times 60 \times 4 ! .5 \times 60)+\theta_{\mathrm{s}}^{2}}{(2 ! .1 \times 60)^{2}+\theta_{\mathrm{s}}^{2}}$,

where $\theta_{\mathrm{s}}$ is the effective circular Gaussian source size in arcseconds. For the central position the ratio of the Odin and SWAS ${ }^{13} \mathrm{CO}$ peak antenna temperatures is $\sim 2.5$ which is equivalent to a mean source size of $\sim 100^{\prime \prime}$.

A second approach to estimate the source size is performed by plotting the antenna temperatures in our Odin strip map vs. position, and fitting a Gaussian. After deconvolution with the $126^{\prime \prime}$ Odin beam, the resulting one dimensional source size is about $106^{\prime \prime}$. Together with the mean source size of $\sim 100^{\prime \prime}$, this implies a relatively circular source.

The excitation temperature can be estimated by plotting a rotation diagram of several transitions. The level populations are given by the Boltzmann distribution and the excitation temperatures for all the energy levels are assumed to be the same. The beam-filling corrected integrated intensities of all lines are plotted as a function of the upper state energy in a semi-log plot based upon the relation (Persson et al. 2007)

$\ln \frac{8 \pi k v_{u l}^{2}}{h c^{3} g_{u} A_{u l}} \int T_{\mathrm{b}} \mathrm{d} v=\ln \frac{N_{\mathrm{ROT}}}{Q(T)}-\frac{E_{u}}{k T_{\mathrm{ex}}}$,

where $k$ is the Boltzmann constant, $v_{u l}$ is the frequency of the transition, $h$ is the Planck constant, $c$ is the speed of light, $A_{u l}$ is the Einstein $A$-coefficient for the transition, $T_{\mathrm{b}}$ is the brightness temperature (observed antenna temperature corrected for beamefficiency and beam-filling), $g_{u}$ and $E_{u}$ are the statistical weight and energy of the upper state, respectively, $Q(T)$ is the partition function, $T_{\mathrm{ex}}$ is the excitation temperature for the transition, and $N_{\text {ROT }}$ is the total column density of the species obtained from the rotational diagram. As customary the frequency axis $v$ has been converted to a velocity axis $v$ using the speed of light. A least-squares fit to the data will produce a straight line with slope $-1 / T_{\text {ROT }}$. If we extrapolate the line to $E_{u}=0 \mathrm{~K}$, the total column density is found from the intersection of the $y$-axis.

Our rotation diagram of the broad outflow ${ }^{13} \mathrm{CO}$ component in the central position (on-line Fig. B.2), including the Onsala 20-m $J=1-0$ transition, the $J=2-1$ and 3-2 transitions from Minchin et al. (1993), and the Odin $J=5-4$ transition, produces $T_{\mathrm{ROT}}=24 \pm 2 \mathrm{~K}$ and $N_{\mathrm{ROT}}=(2.5 \pm 0.4) \times 10^{16} \mathrm{~cm}^{-2}$. All antenna temperatures are corrected with respective beamfilling and beam-efficiencies. This temperature is in agreement with the previously estimated lower limit of the outflow excitation temperature of about 15-20 K (Minchin et al. 1993). The low temperature of the outflow indicates that the emission arises from gas far behind the shock front where the gas has already cooled (Snell et al. 1984b). 




Fig. 2. Comparison of the $\mathrm{CO}(5-4)$ (thick magenta line) and ${ }^{13} \mathrm{CO}(5-4)$ (thin black line) line profiles in a strip map from NE to SW across the PDR.

The excitation temperature of the narrow ${ }^{13} \mathrm{CO}$ PDR emission in the central position is also estimated by means of a rotation diagram (on-line Fig. B.3), including the above mentioned transitions with addition of the $J=6-5$ line from Graf et al. (1993), but excluding $J=1-0$ which seems to originate only in the colder gas component. This produces $T_{\text {ROT }}=69 \pm 27 \mathrm{~K}$, in agreement with Minchin et al. (1993) who find a PDR excitation temperature of about $70 \mathrm{~K}$ at IRS and a steep rise in temperature to about $250 \mathrm{~K}$ at the dissociation front $60^{\prime \prime}-80^{\prime \prime} \mathrm{SW}$ of IRS1. Our PDR column density, $N_{\text {ROT }}\left({ }^{13} \mathrm{CO}\right)=(3.2 \pm 1.8) \times 10^{16} \mathrm{~cm}^{-2}$, is relatively uncertain but in agreement with the RADEX result in Sect. 4, $N\left({ }^{13} \mathrm{CO}\right)=2.8 \times 10^{16} \mathrm{~cm}^{-2}$, and with Plume et al. (1994), $3.4 \times 10^{16} \mathrm{~cm}^{-2}$.

\subsection{Water}

The ortho-water $1_{1,0}-1_{0,1}$ ground-state rotational transition is detected in all positions except outside the bright rim (position 5). Similar to $\mathrm{CO}$ and ${ }^{13} \mathrm{CO}$, a narrow component $\left(\Delta v \sim 3 \mathrm{~km} \mathrm{~s}^{-1}\right.$ ) superimposed on a broader outflow component $\left(\Delta v \sim 8.5 \mathrm{~km} \mathrm{~s}^{-1}\right)$ is observed with emission originating around the central position. Like $\mathrm{CO}$, the water line profile is dominated by outflow emission, clearly seen in a two Gaussian component fit in the on-line Fig. B.6. This is in contrast to ${ }^{13} \mathrm{CO}$ which is dominated by the narrow component (on-line Fig. B.4). The water outflow emission, in addition, mainly shows a red wing.

The non-detection of $\mathrm{H}_{2}^{18} \mathrm{O}$ can be used to set upper limits on the opacity of the main water line using Eq. (1). However, the



Fig. 3. Comparison of the $\mathrm{H}_{2} \mathrm{O} \quad 1_{1,0}-1_{0,1}$ (thick magenta line) and ${ }^{13} \mathrm{CO}(5-4)$ (thin black line) line profiles in a strip map from NE to SW across the PDR.

calculation is not as straightforward as for CO. Because of the high critical density (the ratio of the spontaneous de-excitation and collision coefficients) of the $\mathrm{H}_{2} \mathrm{O} 557 \mathrm{GHz}$ line, the emission of this transition is highly sub-thermal and has a high opacity even for rather low abundances. This high optical depth allows the excitation temperature to be enhanced by radiative trapping. As a result, the corresponding lines of $\mathrm{H}_{2} \mathrm{O}$ and $\mathrm{H}_{2}^{18} \mathrm{O}$ have quite different values of excitation temperature. Their excitation temperatures will also be much lower than the kinetic temperature. Using the RADEX code in Sect. 4 for a kinetic temperature of $55 \mathrm{~K}$ and a molecular hydrogen density $n\left(\mathrm{H}_{2}\right)=4 \times 10^{5} \mathrm{~cm}^{-3}$, we find excitation temperatures of 8.2 and $6.3 \mathrm{~K}$ for the $1_{1,0}-1_{0,1}$ transitions of $\mathrm{H}_{2} \mathrm{O}$ and $\mathrm{H}_{2}^{18} \mathrm{O}$, respectively. If the abundance ratio $\left[{ }^{16} \mathrm{O} /{ }^{18} \mathrm{O}\right]=330$ in water (Persson et al. 2007) and if the two isotopologues are distributed similarly, then the $5 \sigma$ upper limit on the intensity of the $\mathrm{H}_{2}^{18} \mathrm{O}$ line implies that the optical depth of the $\mathrm{H}_{2} \mathrm{O}$ line is $\$ 60$. If the assumed density is lower by a factor of 10 , the difference in excitation temperatures will increase and change the upper limit to $\lesssim 120$.

The high $\mathrm{H}_{2} \mathrm{O}$ opacity, in addition, often produces selfabsorbed spectra. Our observations, however, do not show any obvious signs of self-absorption in contrast to our $\mathrm{CO}$ observations. When comparing the centre velocities of the narrow components of water and $\mathrm{CO}$ to ${ }^{13} \mathrm{CO}$, we find that the velocities of water and ${ }^{13} \mathrm{CO}$ are well matched (Fig. 3 and Tables B.2, B.3), while $\mathrm{CO}$ and ${ }^{13} \mathrm{CO}$ in positions $1-4$ are not (Fig. 2). This supports our conclusion that there is no severe self-absorption in $\mathrm{H}_{2} \mathrm{O}$ in contrast to observations of other sources in which 


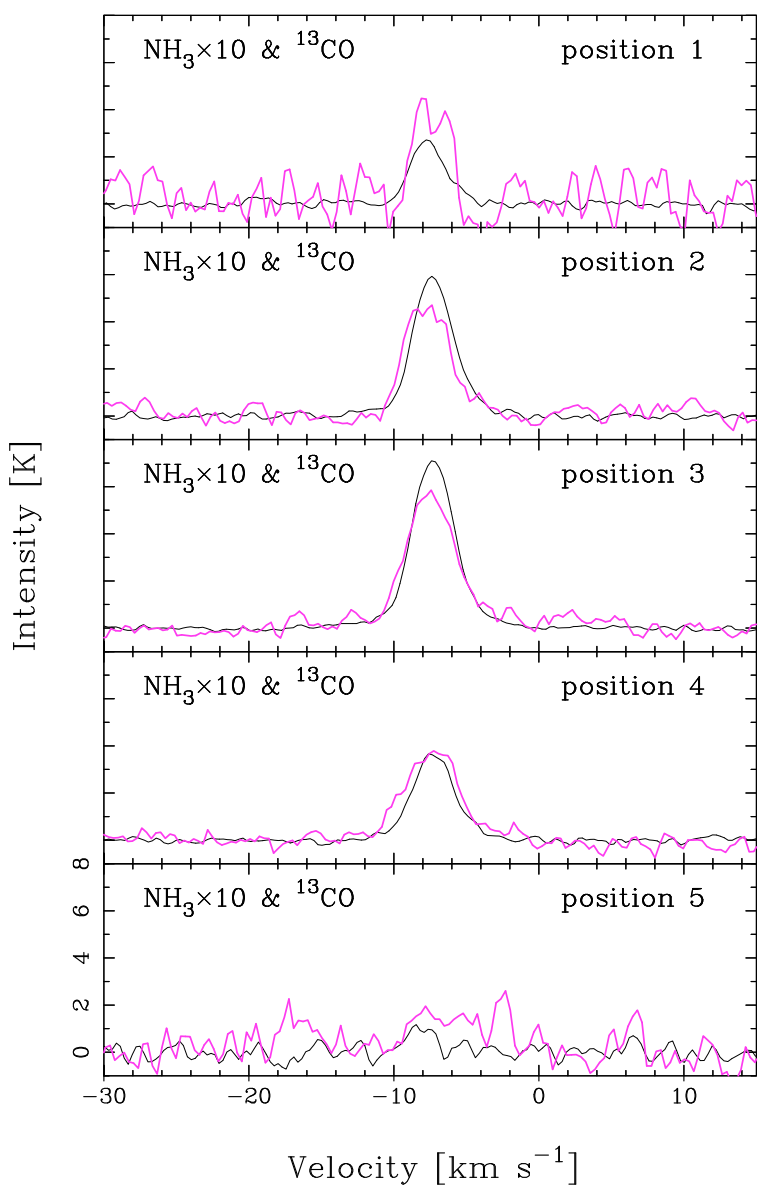

Fig. 4. Comparison of the $\mathrm{NH}_{3} \quad 1_{0}-0_{0}$ (thick magenta line) and ${ }^{13} \mathrm{CO}(5-4)$ (thin black line) line profiles in a strip map from NE to $\mathrm{SW}$ across the PDR.

corresponding self-absorptions were seen in $\mathrm{CO}(7-6)$ and in ISO measurements of $\mathrm{H}_{2} \mathrm{O}$ (Boonman et al. 2003).

The lack of self-absorption points to a rather constant excitation across the region (cf. Snell et al. 1984a). It also implies a lower water abundance in the blue than in the red outflow. A higher water abundance in the red wing may be caused by outflowing gas pushing into the ambient high density cloud thereby producing water. The blue outflow, on the other hand, is leaving the molecular cloud, and expands into an ionized region with low density gas with no or very low water production as a result. This is in agreement with the results in Franklin et al. (2008) who investigated water abundance in molecular outflows with SWAS and find about five times higher abundance in the red outflow compared to the blue.

The sizes of the emitting regions are estimated in the same way as for ${ }^{13} \mathrm{CO}$ (Eq. (4)). For the central position the ratio of the Odin and SWAS peak antenna temperatures is $\sim 2.4$, equivalent to a mean source size of $\sim 100^{\prime \prime}$. This confirms previous suggestions that the water emission does not fill the SWAS beam (Boonman et al. 2003). To obtain estimates of the size of each component, we use the amplitudes of the fitted Gaussians from the Odin and SWAS spectra. The ratios of the PDR and outflow components are $\sim 2.2$ and $\sim 2.6$, which corresponds to mean source sizes of $120^{\prime \prime}$ and $85^{\prime \prime}$, respectively.

Gaussian fits to the amplitudes in each position in the Odin strip map results in one-dimensional source sizes of $\gtrsim 150^{\prime \prime}$ and $\$ 50^{\prime \prime}$ for the PDR and outflow, respectively. Taken together, these two calculations of source sizes suggest that the water

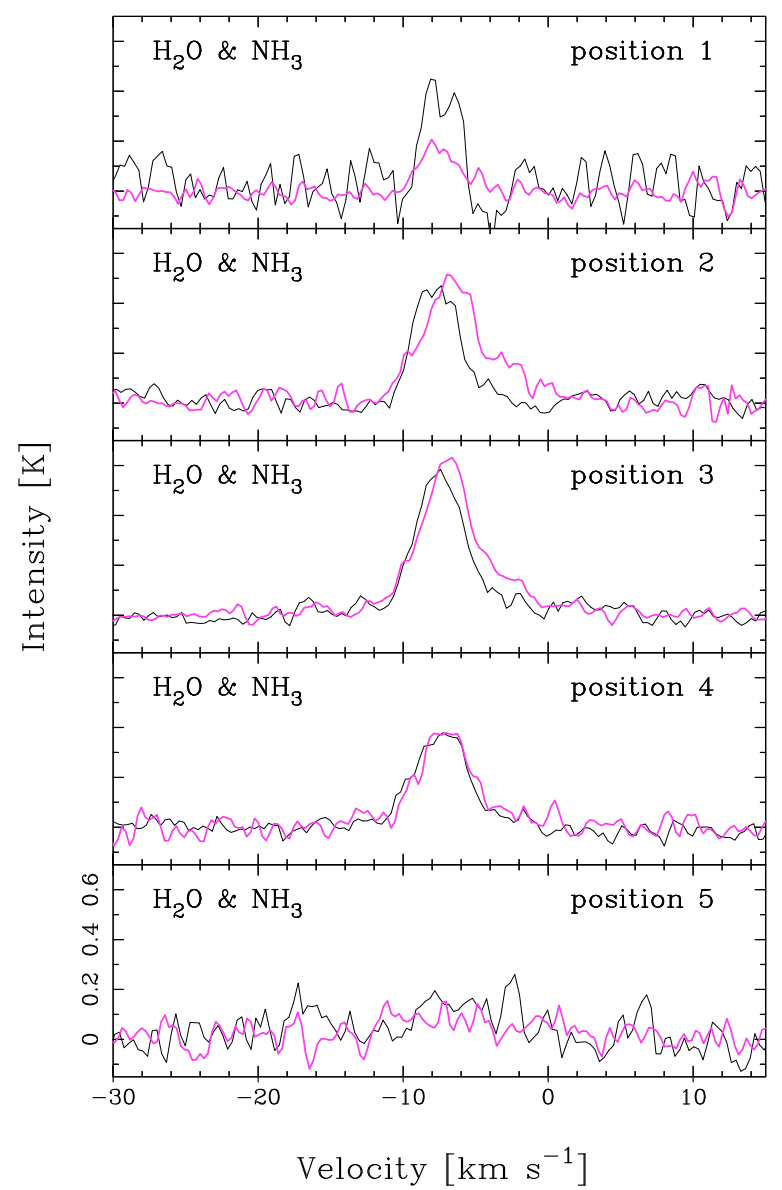

Fig. 5. Comparison of the $\mathrm{H}_{2} \mathrm{O}$ (thick magenta line) $1_{1,0}-1_{0,1}$ and the $\mathrm{NH}_{3}$ (thin black line) $1_{0}-0_{0}$ line profiles in a strip map from NE to SW across the PDR.

emission from the PDR is more extended in the NE-SW direction (same direction as our strip map), while the contrary applies to the outflow, indicating a NW-SE elongation.

A third approach to estimate the beam-filling can be achieved by using the radiative transfer equation. With a constant source function, the solution of the radiative transport equation is

$T_{\mathrm{A}}^{*}=T_{\mathrm{b}} \eta_{\mathrm{mb}} \eta_{\mathrm{bf}}=\left(J\left(T_{\mathrm{ex}}\right)-J\left(T_{\text {cont }}\right)\right)\left(1-\mathrm{e}^{-\tau}\right) \eta_{\mathrm{mb}} \eta_{\mathrm{bf}}$,

where $J\left(T_{\text {cont }}\right)$ is the background continuum radiation.

For an optically thick line and neglecting the background radiation

$T_{\mathrm{A}}^{*} \approx J\left(T_{\mathrm{ex}}\right) \eta_{\mathrm{mb}} \eta_{\mathrm{bf}}$.

If $T_{\text {ex }}$ is known, then the beam-filling can be estimated. Using $T_{\mathrm{ex}}=8.2 \mathrm{~K}$ obtained from RADEX and Eq. (7) give a beam-filling factor that corresponds to a PDR source size of about $110^{\prime \prime}$, which is close to the source size of $120^{\prime \prime}$ obtained from the relative temperatures from Odin and SWAS. This implies that the gas is somewhat clumped, and that the surface filling fraction of the high density PDR gas is $\sim 84 \%$. The clumpy structure of the cloud is confirmed by previous observations (Snell et al. 1984a,b; Zhou et al. 1993; Minchin et al. 1993; Hayashi \& Murata 1992; Ashby et al. 2000a) and our model results in Sect. 5. The amount of surface clumping is, however, not possible to determine very accurately due to the uncertainty of the excitation temperature which is affected by the assumed density. With $T_{\mathrm{ex}}=9 \mathrm{~K}$ and $10 \mathrm{~K}$ the same calculations give source sizes 
of $86^{\prime \prime}$ and $69^{\prime \prime}$, with corresponding surface filling fractions of $50 \%$ and $33 \%$, respectively. Including the background radiation in Eq. (6) also introduces additional uncertainties.

\subsection{Ammonia}

A complex energy level diagram with a wealth of transitions covering different temperatures and densities has made ammonia a very valuable diagnostic of physical conditions in the interstellar medium. The $1_{0}-0_{0}$ ground-state rotational transition, observed by Odin, has an upper state energy comparable to the extensively observed inversion transitions (e.g. Ungerechts et al. 1986; Zhou et al. 1993), but the critical density is about four orders of magnitude larger. It is therefore likely that these transitions partly probe different gas components. The $1_{0}-0_{0}$ transition has a high opacity and is highly sub-thermally excited in most cases like our observed water transition.

The $1_{0}-0_{0}$ transition of ortho- $\mathrm{NH}_{3}$ is detected in all positions except outside the bright rim (position 5). The line profiles and centre velocities are very similar to those of ${ }^{13} \mathrm{CO}(5-4)$ with an exception in position 1 (Fig. 4). Our comparison of ammonia and water in Fig. 5 shows an almost identical line profile at the bright rim (position 4), which suggests emission from the same gas and velocity fields at this position originating in the PDR. In the central position, the water line profile is dominated by the outflow in contrast to ammonia. Both species displays, however, very similar emission in the blue outflow, while water has a more pronounced outflow in the red wing. The water emission from the outflow seems to be almost the same in position 2 and 3 , while the ammonia emission from the outflow decreases in position 2. This suggests that the outflow production mechanism is more efficient for water than ammonia.

Deepest in the molecular cloud a very weak water line is seen. The ammonia emission is, however, considerably stronger than the water emission at this position. This confirms previous observations of $\mathrm{NH}_{3}$ inversion transitions (Zhou et al. 1993; Ungerechts et al. 1986) who find an $\mathrm{NH}_{3}$ peak further into the cloud at about 1 ' northeast of IRS1. When we plot the peak antenna temperatures vs. position it is obvious that we see additional emission in position 1. A Gaussian fit to positions 2-4 gives a source size of $\sim 130^{\prime \prime}$, with a peak about $10^{\prime \prime}$ into the cloud from the centre. This size is relatively uncertain due to a fit to only three positions, but agrees well with the source sizes estimated from ${ }^{13} \mathrm{CO}$ and $\mathrm{H}_{2} \mathrm{O}$.

The Odin observations indicate that in addition to the warm, dense PDR and a weak outflow around IRS, we also observe $\mathrm{NH}_{3}$ from the same cold and extended gas deep in the cloud (position 1 ) as ${ }^{13} \mathrm{CO}(1-0)$ at a temperature about $20-30 \mathrm{~K}$. This is also in agreement with Ungerechts et al. (1986) and Zhou et al. (1993) who find an $\mathrm{NH}_{3}$ rotation temperature of $40 \mathrm{~K}$ in between our first and second position. Chemical models predict that $\mathrm{NH}_{3}$ is not depleted in high density regions $\left(\$ 1 \times 10^{6} \mathrm{~cm}^{-3}\right)$ and is therefore relatively more abundant than other molecules such as water and CO (Bergin \& Langer 1997; Bergin 2003).

The surface filling fraction of the narrow PDR component of ammonia is also estimated using the radiative transfer equation (Eq. (7)). The excitation temperature is approximately the same as for water thereby producing similar surface filling factors. Together with the similarity of the line profiles, source sizes and centre velocities of the $\mathrm{H}_{2} \mathrm{O}, \mathrm{NH}_{3}$ and ${ }^{13} \mathrm{CO}(5-4)$ narrow components, this points to emission from the same high density PDR clumps with the same temperature of about $70 \mathrm{~K}$ for all species (cf. Boonman et al. 2003).

\subsection{Outflow abundances}

The collisional de-excitation of the ground-state rotational transition of ortho-water is very low in the density ranges considered here due to the high critical density of $\sim 3 \times 10^{8} \mathrm{~cm}^{-3}$ at a temperature of $40 \mathrm{~K}$. A simple analytic expression for the antenna temperature of a two-level system in the low collision rate limit can therefore be used since collisional excitation always results in a photon that escapes the cloud. Thus, even though our transition may be optically thick, it is effectively thin (Linke et al. 1977; Snell et al. 2000b). We use this approach to estimate the water abundance in the outflow (Snell et al. 2000b)

$$
\int T_{\mathrm{b}} \mathrm{d} v=C n_{\mathrm{H}_{2}} \frac{c^{3}}{2 v^{3} k} N\left(\mathrm{o}-\mathrm{H}_{2} \mathrm{O}\right) \frac{h v}{4 \pi} \exp \left(-h v / k T_{K}\right),
$$

where $C$ is the collision rate which equals the product of the cross-section and velocity. Dividing Eq. (8) with $N\left(\mathrm{H}_{2}\right)$ and scaling the calculated factor in Snell et al. (2000b) to our derived rotational outflow temperature of $24 \mathrm{~K}$ (with $\sqrt{T}$ ) produces the fractional abundance of ortho-water with respect to $\mathrm{H}_{2}$ in the outflow

$$
X\left(\mathrm{o}-\mathrm{H}_{2} \mathrm{O}\right)=5.1 \times 10^{19} \frac{\int T_{\mathrm{b}} \mathrm{d} v}{N\left(\mathrm{H}_{2}\right) n_{\mathrm{H}_{2}}} .
$$

The $\mathrm{H}_{2}$ column density in the outflow is calculated from $N_{\mathrm{ROT}}\left({ }^{13} \mathrm{CO}\right)$ of the broad component. Using typical values of $\left[\mathrm{CO} / \mathrm{H}_{2}\right]=1 \times 10^{-4}$ and $\left[{ }^{12} \mathrm{C} /{ }^{13} \mathrm{C}\right]=70$ we obtain $N\left(\mathrm{H}_{2}\right)=1.8 \times 10^{22} \mathrm{~cm}^{-2}$. Together with a range of mean molecular hydrogen densities of $5 \times 10^{4}-5 \times 10^{5} \mathrm{~cm}^{-3}$ we obtain a total water outflow abundance of $\sim 4 \times 10^{-8}-4 \times 10^{-7}$. This is in agreement with Franklin et al. (2008) who found an outflow abundance in the blue wing of $2.4 \times 10^{-8}$ and $1.0 \times 10^{-7}$ in the red wing using a kinetic temperature of $50 \mathrm{~K}$ and a molecular density of $10^{5} \mathrm{~cm}^{-3}$.

The ammonia abundance in the outflow is estimated using the Local Thermal Equilibrium (LTE) approximation. Assuming LTE and adding corrections for opacity and beam-filling, the total source averaged column density can be calculated as

$N_{\mathrm{LTE}}=\frac{C_{\tau}}{\eta_{\mathrm{bf}}} \frac{8 \pi k v_{u l}^{2}}{h c^{3}} \frac{1}{A_{u l}} \frac{Q(T)}{g_{u}} \exp \left(E_{u} / k T_{\mathrm{ex}}\right) \int T_{\mathrm{b}} \mathrm{d} v$

where $C_{\tau}=\tau /(1-\exp (-\tau))$ is the opacity correction factor. Using $C_{\tau}=1$, a source size of $85^{\prime \prime}$ to calculate $\eta_{\mathrm{bf}}$ and an excitation temperature of $10 \mathrm{~K}$, the ammonia outflow column density is estimated to be $\sim 1.2 \times 10^{13} \mathrm{~cm}^{-2}$. This column density is thus not opacity-corrected and may therefore be underestimated. The ammonia outflow abundance is then calculated as $X\left(\mathrm{NH}_{3}\right)=N_{\mathrm{NH}_{3}} / N_{\mathrm{H}_{2}}$ and is found to be $\sim 7 \times 10^{-10}$.

\section{Model results: RADEX - a homogeneous model}

In this section we use an enhanced version of the RADEX ${ }^{2}$ (van der Tak et al. 2007) code to compute the intensities of the narrow components of the transitions observed by Odin, and the intensities of several atomic and molecular transitions that previously have been observed in S140. The RADEX codex applies a very simple method of mean escape probabilities to the radiative transfer and yields results that are similar to those in

\footnotetext{
2 The published version will soon include these enhancements, see http://www . sron.rug.nl/ vdtak/radex/index. shtml
} 
Table 2. Observations and model calculations for the narrow PDR component in the RADEX homogeneous model ${ }^{a}$.

\begin{tabular}{|c|c|c|c|c|c|c|c|c|c|c|}
\hline \multirow[t]{2}{*}{ Species } & \multirow[t]{2}{*}{ Transition } & \multirow{2}{*}{$\begin{array}{r}\text { Frequency } \\
v \\
{[\mathrm{GHz}]}\end{array}$} & \multicolumn{3}{|c|}{ "Observations } & \multirow[t]{2}{*}{ Ref. } & \multicolumn{4}{|c|}{ "Model calculations } \\
\hline & & & $\begin{array}{r}T_{\mathrm{b}} \\
{[\mathrm{K}]}\end{array}$ & $\begin{array}{c}4 \pi I \\
{\left[\mathrm{erg} \mathrm{s}^{-1} \mathrm{~cm}^{-2}\right]}\end{array}$ & $\begin{array}{c}\Delta v \\
{\left[\mathrm{~km} \mathrm{~s}^{-1}\right]}\end{array}$ & & $\begin{array}{l}T_{\mathrm{ex}} \\
{[\mathrm{K}]}\end{array}$ & $\tau$ & $\begin{array}{r}T_{\mathrm{b}} \\
{[\mathrm{K}]}\end{array}$ & $\begin{array}{c}4 \pi I \\
{\left[\mathrm{erg} \mathrm{s}^{-1} \mathrm{~cm}^{-2}\right]}\end{array}$ \\
\hline $\mathrm{H}_{2} \mathrm{O}$ & $1_{1,0}-1_{0,1}$ & 556.936 & 0.92 & $\ldots$ & 3.1 & $c$ & 8.2 & 6.6 & 0.92 & $6.8 \times 10^{-6}$ \\
\hline $\mathrm{H}_{2}^{18} \mathrm{O}$ & $1_{1,0}-1_{0,1}$ & 547.676 & $<0.051$ & $\ldots$ & $(3.1)^{b}$ & $c$ & 6.3 & 0.2 & $<0.05$ & $<3.5 \times 10^{-7}$ \\
\hline \multirow[t]{5}{*}{$\mathrm{NH}_{3}$} & $1_{0}-0_{0}$ & 572.498 & 1.1 & $\ldots$ & 3.3 & $c$ & 9.0 & 6.1 & 1.1 & $9.2 \times 10^{-6}$ \\
\hline & $(1,1)$ & 23.695 & 2.9 & $\ldots$ & $\ldots$ & $d$ & 54 & 0.02 & 0.87 & $2.5 \times 10^{-10}$ \\
\hline & $(2,2)$ & 23.723 & 2.0 & $\ldots$ & $\ldots$ & $d$ & 47 & 0.01 & 0.50 & $1.7 \times 10^{-10}$ \\
\hline & $(3,3)$ & 23.870 & 1.4 & $\ldots$ & $\ldots$ & $d$ & -5.1 & -0.25 & 2.2 & $9.0 \times 10^{-10}$ \\
\hline & $(4,4)$ & 24.139 & 0.15 & $\ldots$ & $\ldots$ & $d$ & 46 & 0.001 & 0.06 & $3.8 \times 10^{-11}$ \\
\hline \multirow[t]{2}{*}{${ }^{13} \mathrm{CO}$} & $2-1$ & 220.399 & 12 & $\ldots$ & 3.3 & $e$ & 55 & 0.2 & 9.1 & $4.3 \times 10^{-6}$ \\
\hline & $5-4$ & 550.926 & 14.7 & $\ldots$ & 3.2 & $c$ & 51 & 0.5 & 14.7 & $1.1 \times 10^{-4}$ \\
\hline \multirow[t]{4}{*}{ CS } & $2-1$ & 97.981 & 8.6 & $\ldots$ & 3.0 & $f$ & 47 & 0.2 & 9.1 & $3.5 \times 10^{-7}$ \\
\hline & $3-2$ & 146.969 & 10.2 & $\ldots$ & 3.0 & $f$ & 20 & 1.0 & 10.0 & $1.3 \times 10^{-6}$ \\
\hline & $5-4$ & 244.936 & 5.4 & $\ldots$ & 3.0 & $f$ & 13 & 1.3 & 5.5 & $3.3 \times 10^{-6}$ \\
\hline & $6-5$ & 293.912 & 3.3 & $\cdots$ & 2.2 & $f$ & 12 & 0.7 & 3.1 & $3.3 \times 10^{-6}$ \\
\hline$[\mathrm{C} \mathrm{I}]$ & $1-0$ & 492.161 & 3.7 & $\cdots$ & 3.5 & $g$ & 54 & 0.1 & 3.7 & $2.1 \times 10^{-5}$ \\
\hline [C II] & $3 / 2-1 / 2$ & 1900.537 & $\ldots$ & $4.4 \times 10^{-3}$ & $\ldots$ & $h$ & 55 & 1.0 & 13.4 & $4.4 \times 10^{-3}$ \\
\hline$[\mathrm{O}$ I] & $1-2$ & 4744.778 & $\begin{array}{l}\cdots \\
\cdots\end{array}$ & $4.1 \times 10^{-3}$ & $\begin{array}{l}\cdots \\
\cdots\end{array}$ & $h$ & 46 & 0.9 & 0.88 & $4.5 \times 10^{-3}$ \\
\hline
\end{tabular}

${ }^{a}$ All results for $T_{\mathrm{K}}=55 \mathrm{~K}, n\left(\mathrm{H}_{2}\right)=4 \times 10^{5} \mathrm{~cm}^{-3} . T_{\mathrm{b}}=$ Rayleigh-Jeans brightness temperature, $4 \pi I=$ flux, $\Delta v=$ line width, $T_{\mathrm{ex}}=$ excitation temperature, $\tau=$ line-centre peak opacity; ${ }^{b}$ from $\mathrm{H}_{2} \mathrm{O} ;{ }^{c}$ this work; ${ }^{d}$ Ungerechts et al. (1986); ${ }^{e}$ Minchin et al. (1993); ${ }^{f}$ Snell et al. (1984a); ${ }^{g}$ Plume et al. (1994); ${ }^{h}$ Emery et al. (1996).

the large-velocity-gradient approximation, assuming an isothermal and homogeneous medium, but without an implicit assumption of a gradient in velocity. Although much more sophisticated methods of radiative transfer have been used to construct models of the atomic and molecular line emission from this region, it is useful to examine a simple, internally consistent model of a homogeneous cloud that reproduces the principal observed facts. Such a model can be used in particular to assess the role of radiative coupling of molecular excitation to the intense continuum radiation within the PDR. In Sect. 5, these results are compared to the results from a 3D inhomogeneous PDR model that calculates the temperature and abundance distributions, and a multizone escape probability method that calculates the level populations and intensity distributions of water and ammonia (Poelman \& Spaans 2005, 2006).

The molecular data files for $\mathrm{H}_{2} \mathrm{O}$ and $\mathrm{NH}_{3}$ have been enlarged, compared with those previously available through the Leiden Atomic and Molecular Database (LAMDA) ${ }^{3}$. Data for the ortho and para forms of these molecules have been combined into a single file and infrared transitions have been added. Collision rates published by Faure et al. (2007) for $\mathrm{H}_{2}+\mathrm{H}_{2} \mathrm{O}$ and by Faure et al. (2004) for $\mathrm{e}^{-}+\mathrm{H}_{2} \mathrm{O}$ are now used. Two different data files have been used to analyse the excitation of $\mathrm{NH}_{3}$. The smaller includes only the lowest 112 levels of the vibrational ground state and 140 radiative transitions between them. A larger file, derived mainly from the HITRAN ${ }^{4}$ database, has also been tested: it contains 2392 levels and 15067 radiative transitions, including lines of several $v_{2}$ and $v_{4}$ vibrational bands in the mid-infrared.

We include a simple dust model of the broad-band spectrum (Thronson et al. 1983; Minchin et al. 1995; Ney \& Merrill 1980) at submm and far-infrared wavelengths in order to characterise the internal radiation sensed by the molecules (a detailed description is found in Appendix A of the on-line material).

The principal parameters needed to specify a model are the kinetic temperature $T_{\mathrm{K}}$ and the average number density

\footnotetext{
3 http://www.strw.leidenuniv.nl/ moldata

${ }^{4}$ http://cfa-www.harvard.edu/hitran
}

of molecular hydrogen $n\left(\mathrm{H}_{2}\right)$. Our adopted dust model implies $N\left(\mathrm{H}_{2}\right)=4.7 \times 10^{22} \mathrm{~cm}^{-2}$ and an average density $n\left(\mathrm{H}_{2}\right)=3 \times 10^{4} \mathrm{~cm}^{-3}$ in a homogeneous, spherical cloud with a source size $L=0.5 \mathrm{pc}$ (derivation in Appendix A, on-line material). Unfortunately, this average density is inconsistent with our observations. Although a uniform RADEX model can be constructed based upon this density, this low-density model cannot explain the observed intensities of the pure rotational lines of $\mathrm{H}_{2} \mathrm{O}$ and $\mathrm{NH}_{3}$ unless the line-centre optical depths are of the order of 200 and 100, respectively. Such large opacities would imply significant line broadening through saturation of the emission, which conflicts with the observed narrow profiles.

High densities of $7 \times 10^{5} \mathrm{~cm}^{-3}$ are found by multitransition CS observations (Snell et al. 1984a), and $5 \times 10^{5} \mathrm{~cm}^{-3}$ from $\mathrm{NH}_{3}$ inversion transitions (Ungerechts et al. 1986). We include the CS transitions in our RADEX model in addition to our own observations, and our best fit suggests a mean molecular density of $4 \times 10^{5} \mathrm{~cm}^{-3}$ and a temperature of $55 \mathrm{~K}$. This suggests that the strongly emitting molecules occupy only a $7 \%$ fraction of the volume. The $\mathrm{H}_{2}$ column density, which is needed to obtain the abundances, $X(x)=N(x) / N\left(\mathrm{H}_{2}\right)$, is calculated from the $N\left({ }^{13} \mathrm{CO}\right)$ obtained with RADEX. This column density is also in agreement with $N_{\mathrm{ROT}}\left({ }^{13} \mathrm{CO}\right)$ found in Sect. 3.1. Using $\left[{ }^{12} \mathrm{C} /{ }^{13} \mathrm{C}\right]=70$ and $\left[\mathrm{CO} / \mathrm{H}_{2}\right]=10^{-4}$ we obtain $N\left(\mathrm{H}_{2}\right)=2.0 \times 10^{22} \mathrm{~cm}^{-2}$ for the narrow PDR component.

Table 2 collects the observed intensities and the corresponding values calculated from the model. A source size of $120^{\prime \prime}$, derived in Sect. 2, is used to correct the antenna temperatures $T_{\mathrm{A}}^{*}$ to Rayleigh-Jeans brightness temperatures $T_{\mathrm{b}}$ according to the scaling in Eq. (6). The estimated abundances and column densities are summarized in Table 3. Note that the best-fitting model of the [C II] $\lambda 157 \mu \mathrm{m}$ line emission implies a density of carbon ions, $n\left(\mathrm{C}^{+}\right)=7.7 \mathrm{~cm}^{-3}$. This is taken to be equal to the electron density, which implies an average electron fraction of $X\left(\mathrm{e}^{-}\right)=1.9 \times 10^{-5}$. This electron fraction is large enough that electron-impact on polar molecules like $\mathrm{H}_{2} \mathrm{O}$ must be competitive with $\mathrm{H}_{2}$ collisions in exciting rotational states. However, the results presented for $\mathrm{NH}_{3}$ so far neglect electron collisions. 
Table 3. Results of the homogeneous RADEX model for the narrow PDR component: column densities and abundances ${ }^{a}$.

\begin{tabular}{lcc}
\hline \hline $\begin{array}{l}\text { Species } \\
x\end{array}$ & $\begin{array}{c}\text { Column Density } \\
N(x) \\
{\left[\mathrm{cm}^{-2}\right]}\end{array}$ & $\begin{array}{c}\text { Abundance } \\
X(x)\end{array}$ \\
\hline $\mathrm{H}_{2} \mathrm{O}$ & $2.0 \times 10^{14}$ & $9.8 \times 10^{-9}$ \\
$\mathrm{H}_{2}^{18} \mathrm{O}$ & $<4.9 \times 10^{12}$ & $<2.5 \times 10^{-10}$ \\
$\mathrm{NH}_{3}$ & $1.5 \times 10^{14}$ & $7.5 \times 10^{-9}$ \\
${ }^{12} \mathrm{CO}$ & $2.0 \times 10^{18}$ & $1.0 \times 10^{-4}$ \\
${ }^{13} \mathrm{CO}$ & $2.9 \times 10^{16}$ & $1.4 \times 10^{-6}$ \\
$\mathrm{CS}$ & $1.5 \times 10^{14}$ & $7.5 \times 10^{-9}$ \\
$\mathrm{C}$ & $2.0 \times 10^{17}$ & $1.0 \times 10^{-5}$ \\
$\mathrm{C}^{+}$ & $9.0 \times 10^{17}$ & $4.5 \times 10^{-5}$ \\
$\mathrm{O}$ & $6.3 \times 10^{17}$ & $3.2 \times 10^{-5}$ \\
\hline
\end{tabular}

a All results for $T_{\mathrm{K}}=55 \mathrm{~K}, n\left(\mathrm{H}_{2}\right)=4 \times 10^{5} \mathrm{~cm}^{-3}, n\left(\mathrm{e}^{-}\right)=7.7 \mathrm{~cm}^{-3}$, $N\left(\mathrm{H}_{2}\right)=2.0 \times 10^{22} \mathrm{~cm}^{-2}$.

The model reproduces nicely all the observations except the ammonia inversion transitions which are predicted to be lower than the observed intensities. The ${ }^{13} \mathrm{CO}(2-1)$ transition is also predicted to be slightly lower than what is observed. These discrepancies may be caused by a co-existing, extended lower excitation gas component, which is not contributing much to the emission of the ${ }^{13} \mathrm{CO}(5-4)$ or $\mathrm{NH}_{3}\left(1_{0}-0_{0}\right)$ lines. Even so, the column densities of $\mathrm{C},{ }^{13} \mathrm{CO}$ and $\mathrm{CS}$ agree well with Plume et al. (1994) and Snell et al. (1984a), and the C abundance is also in agreement with Franklin et al. (2008). The water abundance was previously estimated by Snell et al. (2000a) to be $9 \times 10^{-9}$, and by Ashby et al. (2000a) to be $2 \times 10^{-8}$, both very similar to the abundance calculated by RADEX.

The RADEX model is also tested for higher transitions of $\mathrm{H}_{2} \mathrm{O}$ and shows that some lines are sensitive to various effects. For example, the intensity ratio of the 1661 and $1670 \mathrm{GHz}$ lines appears to be sensitive to the total column density. When the internal radiation field is switched off in the models, the intensities of the transitions involving the lowest rotational levels increase slightly, while the intensities of lines involving more highly excited states are diminished. In particular, the $1661 \mathrm{GHz}$ line is enhanced by radiative excitation and is very sensitive to the total column density. This line will appear in absorption if the opacity in the $557 \mathrm{GHz}$ transition is small since photon-trapping is ineffective at column densities around $10^{14} \mathrm{~cm}^{-2}$ or less.

The excitation of $\mathrm{NH}_{3}$ also shows some interesting effects in these models. Due to the rather high density of $\mathrm{C}^{+}$ions, the reaction of $\mathrm{C}^{+}$with $\mathrm{NH}_{3}$ will destroy ammonia at a rate exceeding $10^{-8} \mathrm{~s}^{-1}$, which is rapid enough to affect the excitation of the metastable levels involved in several of the inversion transitions near $23 \mathrm{GHz}$. Indeed, the calculations indicate that the $(J, K)=(3,3)$ inversion transition is a weak maser (cf. Walmsley \& Ungerechts 1983). In addition, $\mathrm{NH}_{3}$ has strong vibrational transitions in the mid- and near-infrared. When these are included, the intensity of the $572 \mathrm{GHz}$ transition is somewhat suppressed while the lowest inversion transitions are somewhat enhanced.

\section{Model results: clumpy PDR model}

A self-consistent physi-chemical 3D inhomogeneous model is used to compute the temperature of the gas and dust, and the abundance distributions inside the clumpy cloud (detailed descriptions are found in Spaans 1996; Spaans \& van Dishoeck 1997; Poelman \& Spaans 2005). This clumpy PDR model has three free parameters; the volume filling factor $F$ (fraction of
Table 4. Clumpy PDR model parameters.

\begin{tabular}{cllllll}
\hline \hline $\begin{array}{l}\text { Size } \\
{[\mathrm{pc}]}\end{array}$ & $\begin{array}{l}n_{\mathrm{c}}(\mathrm{H}) \\
{\left[\mathrm{cm}^{-3}\right]}\end{array}$ & $\begin{array}{l}n_{\text {ic }}(\mathrm{H}) \\
{\left[\mathrm{cm}^{-3}\right]}\end{array}$ & $\begin{array}{l}F^{a} \\
{[\%]}\end{array}$ & $\begin{array}{l}l_{\mathrm{c}}^{b} \\
{[\mathrm{pc}]}\end{array}$ & $\begin{array}{l}T_{\text {gas }} \\
{[\mathrm{K}]}\end{array}$ & $\begin{array}{l}T_{\text {dust }} \\
{[\mathrm{K}]}\end{array}$ \\
\hline 0.5 & $2 \times 10^{6}$ & $1 \times 10^{4}$ & 8 & 0.03 & $\begin{array}{l}9-40^{c} \\
35-195^{d}\end{array}$ & $18-32^{c}$ \\
\hline
\end{tabular}

${ }^{a}$ Volume filling fraction of clumps; ${ }^{b}$ clump size; ${ }^{c}$ temperatures in clumps; ${ }^{d}$ temperatures in interclump medium.

the cloud that is occupied by clumps), a clump size $l_{\mathrm{c}}$, and the clump-interclump ratio $n_{\mathrm{c}}(\mathrm{H}) / n_{\mathrm{ic}}(\mathrm{H})$. The total hydrogen density $n_{\mathrm{H}}=n(\mathrm{H})+2 n\left(\mathrm{H}_{2}\right)$. In the dense clumps, hydrogen is entirely molecular, thus $n(\mathrm{H})=2 n\left(\mathrm{H}_{2}\right)$. The PDR model parameters used in this paper are given in Table 4 . We use a velocity dispersion of $1.2 \mathrm{~km} \mathrm{~s}^{-1}$ (Zhou et al. 1994) equivalent to a FWHM line width of $2.0 \mathrm{~km} \mathrm{~s}^{-1}$ for an optically thin line. With an opacity of about 5-10 the line is broadened by a factor of $\sim 1.5-2$ to $3-4 \mathrm{~km} \mathrm{~s}^{-1}$, consistent with our observations. The incident radiation field is taken to be $I_{\mathrm{UV}}=140$ with respect to the Draine (1978) field. The clumps are randomly distributed in the interclump medium and the volume filling fraction is taken to be $8 \%$ (Spaans \& van Dishoeck 1997; Ashby et al. 2000a; Poelman \& Spaans 2005). The grid size is $81 \times 81 \times 81$, corresponding to a resolution of $0.006 \mathrm{pc}$ and a total size of $0.5 \mathrm{pc}$ (about $110^{\prime \prime}$ at a distance of $910 \mathrm{pc}$ ).

In the PDR computation, each clump is joined smoothly to the inter-clump medium through a power-law $\propto 1 / R^{2}$ density distribution. This envelope contains very little molecular material and the bulk of the impinging radiation field is always absorbed by the clumps and not the inter-clump medium. The definition of the mean $\mathrm{H}_{2}$ density and the mean molecular abundances $X$ of species $x$ is thus made through $\langle n\rangle=F n_{\mathrm{c}}+(1-F) n_{\mathrm{ic}}$ and $X_{\text {mean }}(x)=F X_{\mathrm{c}}(x)+(1-F) X_{\mathrm{ic}}(x)$ to allow an unambiguous comparison with molecular line observations.

A high molecular hydrogen density of $\gtrsim 1 \times 10^{6} \mathrm{~cm}^{-3}$ in the clumps is required to match the intensities observed by Odin and SWAS. Note that this clump density is considerably higher than that used by Poelman \& Spaans $(2005,2006)$. The high density and clumpy medium suggested by our PDR model is, however, also supported by previous density estimates. For example, density variations between $10^{4}-10^{6} \mathrm{~cm}^{-3}$ are necessary to explain the apparent coincidence of the $\mathrm{CI}$ and $\mathrm{CO}$ ridges as well as the distance between the $\mathrm{CI}$ ridge and the bright rim (Hayashi \& Murata 1992). Emission from numerous, small, dense $\left(10^{5}-2 \times 10^{6} \mathrm{~cm}^{-3}\right)$ and optically thick clumps of gas, where the number density of clumps decreases with distance from the CS cloud centre close to IRS, is the best explanation of the CS column density variations, as well as the observed opacities and temperatures obtained from multitransition observations of CS (Snell et al. 1984a; Zhou et al. 1994). As molecular hydrogen density in the interclump medium we use $5 \times 10^{3} \mathrm{~cm}^{-3}$.

The resulting abundances, with respect to $\mathrm{H}_{2}$, in the clumps and the inter-clump medium, as well as a mean value over the whole region, are found in Table 5. The mean value is about half than found by RADEX. In the clumps, the water abundances vary between $10^{-7}-10^{-9}$ with an average of $4.8 \times 10^{-8}$. The low density in the interclump medium results in low water abundances between $1 \times 10^{-11}-5 \times 10^{-9}$ with an average of $8.9 \times 10^{-10}$. Ammonia is found to have about the same average abundance as water, $4.3 \times 10^{-8}$, in the clumps, while lower by almost an order of magnitude in the inter-clump medium, $1.1 \times 10^{-10}$.

The result from our PDR model is used as input to a three-dimensional multi-zone escape probability method which 
Table 5. Resulting abundances with respect to $n_{\mathrm{H}_{2}}$ for the narrow PDR components of water and ammonia from the clumpy PDR model.

\begin{tabular}{llll}
\hline \hline Species & Comp. & $\begin{array}{l}n_{\mathrm{H}_{2}} \\
{\left[\mathrm{~cm}^{-3}\right]}\end{array}$ & Abundance \\
\hline $\mathrm{H}_{2} \mathrm{O}$ & Interclump & $5.0 \times 10^{3}$ & $8.9 \times 10^{-10}$ \\
& Clump & $1.0 \times 10^{6}$ & $4.8 \times 10^{-8}$ \\
& Mean & $8.5 \times 10^{4}$ & $4.6 \times 10^{-9}$ \\
$\mathrm{NH}_{3}$ & Interclump & $5.0 \times 10^{3}$ & $1.1 \times 10^{-10}$ \\
& Clump & $1.0 \times 10^{6}$ & $4.5 \times 10^{-8}$ \\
& Mean & $8.5 \times 10^{4}$ & $3.7 \times 10^{-9}$ \\
\hline
\end{tabular}

Table 6. Observed and predicted $T_{\mathrm{A}}^{*}$ in the PDR for o- $\mathrm{H}_{2} \mathrm{O}\left(1_{1,0}-1_{0,1}\right)$ with SWAS and Odin at the central position.

\begin{tabular}{lll}
\hline \hline Satellite & $\begin{array}{l}\text { Obs. } T_{\mathrm{A}}^{*} \\
{[\mathrm{mK}]}\end{array}$ & $\begin{array}{l}\text { Pred. } T_{\mathrm{A}}^{*} \\
{[\mathrm{mK}]}\end{array}$ \\
\hline SWAS & 186 & 120 \\
Odin & 416 & 345 \\
\hline
\end{tabular}

computes the level populations of ortho- and para- $\mathrm{H}_{2} \mathrm{O}$ (up to $\sim 350 \mathrm{~K}), \mathrm{CO}$ and $\mathrm{NH}_{3}$, as well as line intensities and opacities (Poelman \& Spaans 2005, 2006). The excitation due to dust emission is fully included. The dust temperature in the model varies between 18 and $32 \mathrm{~K}$. However, due to geometry the highest $T_{\text {dust }}$ is only achieved at the edge of the PDR and the dust component fills only a small part of the region. The bulk of the dust has a temperature of about $25 \mathrm{~K}$ and the dust continuum opacity $\lambda 100 \mu \mathrm{m}$ is 0.02 or smaller. Results from this code differ at most by $10 \%$ compared to other Monte Carlo/Accelerated Lambda Iteration codes. Figure 6 shows the predicted water intensities on a logarithmic scale. Note that the ionizing star is located on the left side of the displayed region. The peak emission is found about $70-80^{\prime \prime}$ from the bright rim, in agreement with our observations of all species.

The resulting model intensities are then used to calculate the expected antenna temperature via

$$
\begin{aligned}
T_{\mathrm{A}}^{*} & =\frac{1}{2} \frac{S A_{\mathrm{eff}}}{k \Delta v}=\frac{1}{2} \frac{b \eta_{\mathrm{bf}} \Omega_{\mathrm{mb}}}{k \Delta v} \frac{\lambda^{2}}{\Omega_{\mathrm{A}}} \\
& =\frac{1}{2} \frac{b \lambda^{2}}{k \Delta v} \eta_{\mathrm{bf}} \eta_{\mathrm{mb}}=\frac{b \lambda^{3}}{2 k \Delta v} \eta_{\mathrm{mb}} \eta_{\mathrm{bf}}[\mathrm{K}],
\end{aligned}
$$

where $S$ is the integrated flux density in $\left[\mathrm{erg} \mathrm{s}^{-1} \mathrm{~cm}^{-2}\right.$ ], the intensity $b$ is in [erg s${ }^{-1} \mathrm{~cm}^{-2} \mathrm{sr}^{-1}$ ], $A_{\text {eff }}$ is the effective antenna area $\eta_{\mathrm{A}} A_{\text {geo }}=\lambda^{2} / \Omega_{\mathrm{A}}\left[\mathrm{cm}^{2}\right], \Delta v$ is the observed line width in $\mathrm{Hz}, \Omega_{\mathrm{mb}}$ is the main beam solid angle in [sr], $\Delta v$ is the observed line width in $\mathrm{cm} \mathrm{s}^{-1}$, and the wavelength $\lambda$ is in $\mathrm{cm}$. We convolve the intensities to the Odin beam, and in addition predict the intensities of the forthcoming observations of the Herschel Space Observatory using a telescope diameter of $350 \mathrm{~cm}$ and a beamefficiency of 0.7. The observed and modelled SWAS and Odin antenna temperatures for the $1_{1,0}-1_{0,1}$ transition are provided in Table 6 for the central position. In addition, the predicted antenna temperatures for Herschel-HIFI of both the ortho- and para- $\mathrm{H}_{2} \mathrm{O} 1_{1,0}-1_{0,1}$ and $1_{1,1}-0_{0,0}$ transitions along a cut from the bright rim are shown in Fig. 7.

The model ortho-water opacities are relatively low with a unweighted mean opacity of 17 over the entire source, with a range between $10^{-5}-800$.

The antenna temperatures from the PDR model agree well with our observations, especially considering uncertainties in source and clump size, as well as the volume filling fraction,

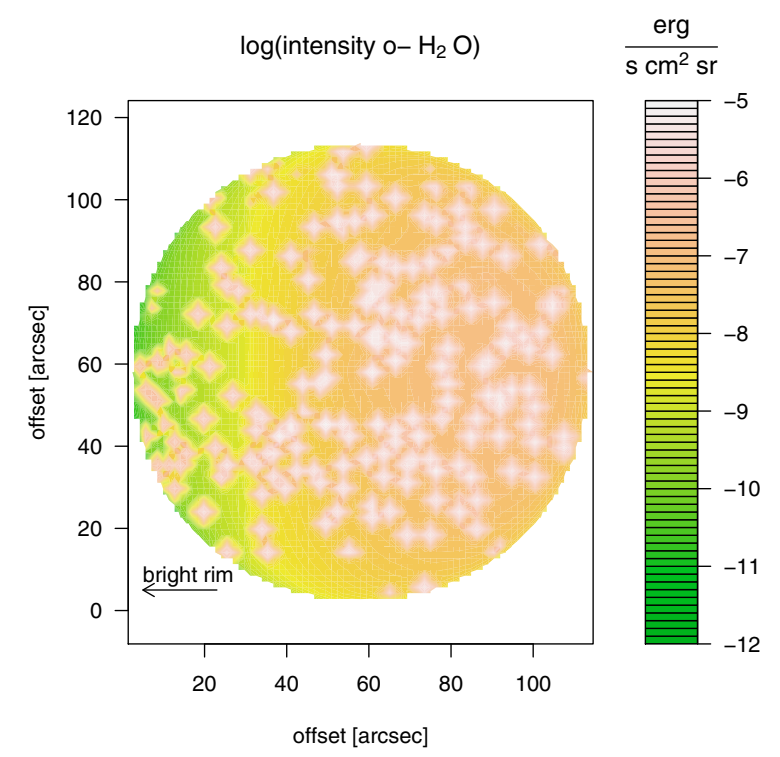

Fig. 6. Result of the PDR high-density model. The water intensity is shown on a logarithmic scale. The ionizing star is located on the left side of the displayed region.

which is the main uncertainty and can be varied to obtain slightly higher or lower $T_{\mathrm{A}}^{*}$. The dust background continuum is also lower than in the RADEX model, which diminishes the effect of radiative excitation of water. A clumpy medium with high clump densities above $10^{6} \mathrm{~cm}^{-3}$ is, however, necessary to produce model antenna temperatures close to the observed values. This is also supported by the resulting low density of $3 \times 10^{4} \mathrm{~cm}^{-3}$ obtained by the dust model in Sect. 4 assuming a homogeneous, spherical cloud, which is about $7 \%$ of the density in our best fitting model.

A clump-interclump density ratio of $\sim 100$ is in general agreement with observations of dense PDRs. Data in CO, sensitive to $n \sim 10^{3-4} \mathrm{~cm}^{-3}$, and high density tracers like CS and HCN, probing $n \sim 10^{5-6} \mathrm{~cm}^{-3}$, often show an extended morphology in CO lines and clump-like features in the CS and HCN gas. Maintaining such a density contrast is a challenge, since the clumps likely evolve on a sound crossing time of $\sim 10^{5} \mathrm{yr}(\sim 0.1 \mathrm{pc}$ clumps). If the interclump medium has a much higher temperature or a higher degree of turbulent motion than the clumps, then a rough pressure equilibrium might hold for times exceeding the sound crossing time, supporting our best-fit density contrast. This would, however, require interclump temperatures of $>10^{3} \mathrm{~K}$ or turbulent motions of $>3 \mathrm{~km} \mathrm{~s}^{-1}$.

\section{Summary and conclusions}

We have used the Odin satellite to observe water, ammonia, and carbon-monoxide in the well-known molecular cloud S140. We have simultaneously observed five-point strip maps across the bright rim in $\mathrm{S} 140$ of the ortho- $\mathrm{H}_{2} \mathrm{O} 1_{1,0}-1_{0,1}$ and the ortho$\mathrm{NH}_{3} 1_{0}-0_{0}$ transitions, as well as $\mathrm{CO}(5-4)$ and ${ }^{13} \mathrm{CO}(5-4)$. The $\mathrm{NH}_{3}$ transition has never previously been observed in $\mathrm{S} 140$. Observations of $\mathrm{H}_{2}^{18} \mathrm{O}$ in the central position resulted in a non-detection at an rms level of $8 \mathrm{mK}$. As support observation we also mapped ${ }^{13} \mathrm{CO}(1-0)$ with the Onsala $20 \mathrm{~m}$ telescope.

Like CO, the water line-profile is dominated by emission from a NW-SE outflow, however, mainly in the red wing. Strong self-absorption is seen in the optically thick $\mathrm{CO}$ emission, while no obvious signs are seen in the ortho $-\mathrm{H}_{2} \mathrm{O}$, ortho $-\mathrm{NH}_{3}$ or the almost optically thin ${ }^{13} \mathrm{CO}$ line profiles. In addition to the outflow, 


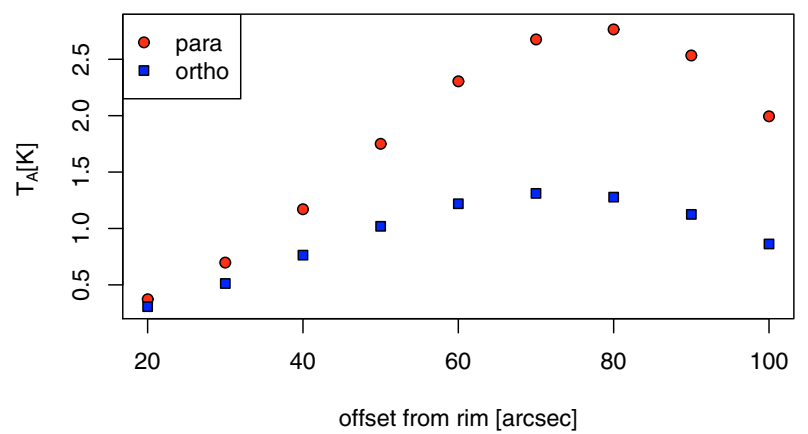

Fig. 7. Predicted antenna temperatures of the ortho- $\mathrm{H}_{2} \mathrm{O}\left(1_{1,0}-1_{0,1}\right)$ and para- $\mathrm{H}_{2} \mathrm{O}\left(1_{1,1}-0_{0,0}\right)$ transitions for the forthcoming Herschel-HIFI observations along a cut $20-100^{\prime \prime}$ from the bright rim.

our water line shows emission from a more extended NE-SW elongated PDR. Both these components originate approximately around our central position. No additional emission is detected closer to the bright rim or further into the molecular cloud where the water most likely is adsorbed onto dust grains. Close to the bright rim the temperature is most likely too high for a detection of our transition with an upper state energy of $61 \mathrm{~K}$. Instead, higher-lying transitions, observable with the Herschel Space Observatory, will have their peak intensity shifted towards the bright rim (Poelman \& Spaans 2005, 2006). Even closer to the bright rim at a few magnitudes of $A_{\mathrm{V}}$, water is, however, photo-dissociated by the UV field.

The ortho $-\mathrm{NH}_{3}$ emission seems to emanate from the same high density clumps in the PDR and the outflow as water, but also shows additional emission further into the cloud where the ambient gas temperature drops to about $30 \mathrm{~K}$. Compared to water in the central position, ammonia has a weaker outflow emission in the red wing although similar in the blue outflow. Close to the bright rim, where the outflow contribution to the emission is very low, the water and ammonia line profiles are almost identical, suggesting an origin in the same gas and velocity fields of the PDR. The ${ }^{13} \mathrm{CO}$ line also shows a very similar line profile as $\mathrm{H}_{2} \mathrm{O}$ and $\mathrm{NH}_{3}$ in this position with a narrow line width of $\sim 3 \mathrm{~km} \mathrm{~s}^{-1}$.

Abundances, with respect to $\mathrm{H}_{2}$, in the PDR are estimated both with an enhanced version of the homogeneous RADEX code and with a clumpy PDR model. This model points to low mean water and ammonia abundances in the PDR of $5 \times 10^{-9}$ and $4 \times 10^{-9}$, respectively. In the high-density clumps both the average water and ammonia abundances increase to $5 \times 10^{-8}$. To match the observed PDR antenna temperatures with Odin and SWAS, a clumpy medium is required by the model with a high molecular hydrogen density in the clumps of $\gtrsim 1 \times 10^{6} \mathrm{~cm}^{-3}$. The resulting RADEX mean abundances are twice as high: $1.0 \times 10^{-8}$ and $8 \times 10^{-9}$ for water and ammonia, respectively, using a molecular hydrogen density of $4 \times 10^{5} \mathrm{~cm}^{-3}$ and a kinetic temperature of $55 \mathrm{~K}$. The differences most likely arise from the uncertainty in density, beam-filling, and volume filling of the clumps. The opacity of the narrow PDR component of the $1_{1,0}-1_{0,1}$ transition is constrained by the narrow line width, and is estimated by RADEX to be $\sim 7$. The PDR model also confirm a low water opacity with an unweighted mean opacity of 17 and a model range of $\sim 10^{-5}-800$. The mean outflow water abundance, derived from a simple two-level approximation, is higher than in the PDR by at least one order of magnitude, $\sim 4 \times 10^{-8}-4 \times 10^{-7}$.

Predictions of antenna temperatures for observations with HIFI are given by our PDR model of the ortho- and para- $\mathrm{H}_{2} \mathrm{O}$ $1_{1,0}-1_{0,1}$ and $1_{1,1}-0_{0,0}$ transitions for nine positions across the bright rim, and are found to peak around $70-80^{\prime \prime}$ from the dissociation front in agreement with our observations.
Acknowledgements. We thank Per Bergman, Per Bjerkeli, Magnus Gustavsson, Matthijs Klomp and also Steve Shore and the organizers of the A\&A and EDP sciences school: Scientific Writing for Young Astronomers for helpful discussions. Generous financial support from the Research Councils and Space Agencies in Sweden, Canada, Finland and France is gratefully acknowledged.

\section{References}

Ashby, M. L. N., Bergin, E. A., Plume, R., et al. 2000a, ApJ, 539, L119 Ashby, M. L. N., Bergin, E. A., Plume, R., et al. 2000b, ApJ, 539, L115 Bergin, E. A. 2003, in SFChem 2002: Chemistry as a Diagnostic of Star Formation, proceedings of a conference held August 21-23, 2002 at University of Waterloo, Waterloo, Ontario, Canada N2L 3G1, ed. C. L. Curry, \& M. Fich (Ottawa, Canada: NRC Press), 63

Bergin, E. A., \& Langer, W. D. 1997, ApJ, 486, 316

Bergin, E. A., Neufeld, D. A., \& Melnick, G. J. 1998, ApJ, 499, 777

Bergin, E. A., Kaufman, M. J., Melnick, G. J., Snell, R. L., \& Howe, J. E. 2003, ApJ, 582, 830

Blair, G. N., Evans, II, N. J., Vanden Bout, P. A., \& Peters, III, W. L. 1978, ApJ, 219, 896

Boonman, A. M. S., \& van Dishoeck, E. F. 2003, A\&A, 403, 1003

Boonman, A. M. S., Doty, S. D., van Dishoeck, E. F., et al. 2003, A\&A, 406, 937

Cernicharo, J., \& Crovisier, J. 2005, Space Sci. Rev., 119, 29

Crampton, D., \& Fisher, W. A. 1974, Publications of the Dominion Astrophysical Observatory Victoria, 14, 283

Draine, B. T. 1978, ApJS, 36, 595

Emery, R., Aannestad, P., Minchin, N., et al. 1996, A\&A, 315, L285 Faure, A., Gorfinkiel, J. D., \& Tennyson, J. 2004, MNRAS, 347, 323

Faure, A., Crimier, N., Ceccarelli, C., et al. 2007, A\&A, 472, 1029

Franklin, J., Snell, R. L., Kaufman, M. J., et al. 2008, ApJ, 674, 1015

Frisk, U., Hagström, M., Ala-Laurinaho, J., et al. 2003, A\&A, 402, L27

Graf, U. U., Eckart, A., Genzel, R., et al. 1993, ApJ, 405, 249

Hayashi, M., \& Murata, Y. 1992, PASJ, 44, 391

Hayashi, M., Hasegawa, T., Omodaka, T., Hayashi, S. S., \& Miyawaki, R. 1987, ApJ, 312, 327

Keene, J., Blake, G. A., \& Phillips, T. G. 1983, ApJ, 271, L27

Larsson, B., Liseau, R., Bergman, P., et al. 2003, A\&A, 402, L69

Larsson, B., Liseau, R., Pagani, L., et al. 2007, A\&A, 466, 999

Linke, R. A., Goldsmith, P. F., Wannier, P. G., Wilson, R. W., \& Penzias, A. A. 1977, ApJ, 214, 50

Liseau, R., Larsson, B., Brandeker, A., et al. 2003, A\&A, 402, L73

Melnick, G. J., Ashby, M. L. N., Plume, R., et al. 2000a, ApJ, 539, L87

Melnick, G. J., Stauffer, J. R., Ashby, M. L. N., et al. 2000b, ApJ, 539, L77

Minchin, N. R., White, G. J., \& Padman, R. 1993, A\&A, 277, 595

Minchin, N. R., Ward-Thompson, D., \& White, G. J. 1995, A\&A, 298, 894

Neufeld, D. A., Lepp, S., \& Melnick, G. J. 1995, ApJS, 100, 132

Ney, E. P., \& Merrill, K. M. 1980, AFGL Report, 80, 50

Nordh, H. L., von Schéele, F., Frisk, U., et al. 2003, A\&A, 402, L21

Olberg, M., Frisk, U., Lecacheux, A., et al. 2003, A\&A, 402, L35

Olofsson, A. O. H., Olofsson, G., Hjalmarson, A., et al. 2003, A\&A, 402, L47

Olofsson, A. O. H., Persson, C. M., Koning, N., et al. 2007, A\&A, 476, 791

Persson, C. M., Olofsson, A. O. H., Koning, N., et al. 2007, A\&A, 476, 807

Plume, R., Jaffe, D. T., \& Keene, J. 1994, ApJ, 425, L49

Poelman, D. R., \& Spaans, M. 2005, A\&A, 440, 559

Poelman, D. R., \& Spaans, M. 2006, A\&A, 453, 615

Preibisch, T., \& Smith, M. D. 2002, A\&A, 383, 540

Rouan, D., Lena, P. J., Puget, J. L., de Boer, K. S., \& Wijnbergen, J. J. 1977, ApJ, 213, L35

Sharpless, S. 1959, ApJS, 4, 257

Snell, R. L., Goldsmith, P. F., Erickson, N. R., Mundy, L. G., \& Evans, II, N. J. 1984a, ApJ, 276, 625

Snell, R. L., Scoville, N. Z., Sanders, D. B., \& Erickson, N. R. 1984b, ApJ, 284, 176

Snell, R. L., Howe, J. E., Ashby, M. L. N., et al. 2000a, ApJ, 539, L101

Snell, R. L., Howe, J. E., Ashby, M. L. N., et al. 2000b, ApJ, 539, L93

Spaans, M. 1996, A\&A, 307, 271

Spaans, M., \& van Dishoeck, E. F. 1997, A\&A, 323, 953

Thronson, Jr., H. A., Lada, C. J., Smith, H. A., et al. 1983, ApJ, 271, 625

Trinidad, M. A., Torrelles, J. M., Rodríguez, L. F., \& Curiel, S. 2007, AJ, 134, 1870

Ungerechts, H., Winnewisser, G., \& Walmsley, C. M. 1986, A\&A, 157, 207

van der Tak, F. F. S., Black, J. H., Schöier, F. L., Jansen, D. J., \& van Dishoeck, E. F. 2007, A\&A, 468, 627

Walmsley, C. M., \& Ungerechts, H. 1983, A\&A, 122, 164

Zhou, S., Evans, II, N. J., Mundy, L. G., \& Kutner, M. L. 1993, ApJ, 417, 613

Zhou, S., Butner, H. M., Evans, II, N. J., et al. 1994, ApJ, 428, 219 


\section{Appendix A: RADEX - construction of a dust model}

In order to relate the molecular column densities, $N(x)$ of species $x$, to fractional abundances, $X(x)=N(x) / N\left(\mathrm{H}_{2}\right)$, a uniform, homogeneous sphere of diameter $L=N\left(\mathrm{H}_{2}\right) / n\left(\mathrm{H}_{2}\right)$ is assumed here. The adopted physical diameter of the PDR corresponding to an angular diameter of $120^{\prime \prime}$ (Sect. 3) at a distance of $910 \mathrm{pc}$ is $0.53 \mathrm{pc}$. This is assumed to be equal to the line-of-sight depth.

The observed intensity of the continuum is used to estimate the internal radiation field sensed by the molecules. We construct a simple model of the broad-band spectrum at submm and far-infrared wavelengths in order both to characterise the internal radiation and to estimate the total column densities of dust and hydrogen. Thronson et al. (1983) measured the far-infrared emission of S140 and found a peak flux density of the order of $10^{4} \mathrm{Jy}$ slightly shortward of $\lambda 100 \mu \mathrm{m}$ in a $49^{\prime \prime}$ beam. Minchin et al. (1995) presented total broad-band fluxes in a $1.5 \times 1.5$ box. We represent the latter results with a two-component model of thermal emission by dust over a solid angle of $\Omega=1.9 \times 10^{-7} \mathrm{sr}$. The main component has a dust temperature $T_{\text {dust }}=40 \mathrm{~K}$ and a long-wavelength $(\lambda>40 \mu \mathrm{m})$ form of the opacity law

$\tau_{\text {dust }}=0.0679(100 / \lambda)^{1.2}$

where $\lambda$ is the wavelength in $\mu \mathrm{m}$. The opacity law is smoothly matched to a standard interstellar extinction law at shorter wavelengths, which is also used to describe the second component at $T_{\text {dust }}=140 \mathrm{~K}$. The opacity of the first dust component corresponds to a visual extinction $A_{\mathrm{V}}=58.8 \mathrm{mag}$. The second component has a smaller optical depth $A_{\mathrm{V}}=0.023 \mathrm{mag}$, but is assumed to cover the same solid angle. In addition, the mid-infrared measurements of Ney \& Merrill (1980) have been adapted in order to specify the radiation field at even shorter wavelengths. In the calculations, the molecules are assumed to be exposed to an average intensity of continuous radiation

$I_{v}=B_{v}\left(T_{\mathrm{CMB}}\right)+\eta \frac{f_{v}^{\text {dust }}}{\Omega}$

where $B_{v}$ is the Planck function, $T_{\mathrm{CMB}}=2.73 \mathrm{~K}$ is the temperature of the cosmic background radiation, $f_{v}^{\text {dust }}$ is the flux density of the 2-component dust model, $\Omega=1.9 \times 10^{-7} \mathrm{sr}$, and $\eta=0.72$ is a dilution factor to scale the brightness of the dust source to the larger beam area of the Odin measurements. It is important to keep in mind that we observe this strong far-infrared radiation; therefore, the co-extensive molecules must sense it also.

For the adopted interstellar extinction law and a standard gas/extinction ratio,

$2 N\left(\mathrm{H}_{2}\right)=1.6 \times 10^{21} A_{\mathrm{V}} \mathrm{cm}^{-2}$,

the adopted dust model implies $N\left(\mathrm{H}_{2}\right)=4.7 \times 10^{22} \mathrm{~cm}^{-2}$ and an average density $n\left(\mathrm{H}_{2}\right)=2.9 \times 10^{4} \mathrm{~cm}^{-3}$ over the source size $L=0.53 \mathrm{pc}$. This average density is, however, inconsistent with the observed molecular line emission in large beams $\left(\theta \geq 1^{\prime}\right)$. Although a uniform RADEX model can be constructed based upon this density, the line-centre optical depths of the pure rotational lines of $\mathrm{H}_{2} \mathrm{O}$ and $\mathrm{NH}_{3}$ would be of the order of 200 and 100, respectively. Such large opacities would imply significant line broadening through saturation of the emission, which conflicts with the observed narrow profiles of $\sim 3 \mathrm{~km} \mathrm{~s}^{-1}$. 
C. M. Persson et al.: Water and ammonia abundances in S140 with the Odin satellite, Online Material p 2

\section{Appendix B: Figures and tables}

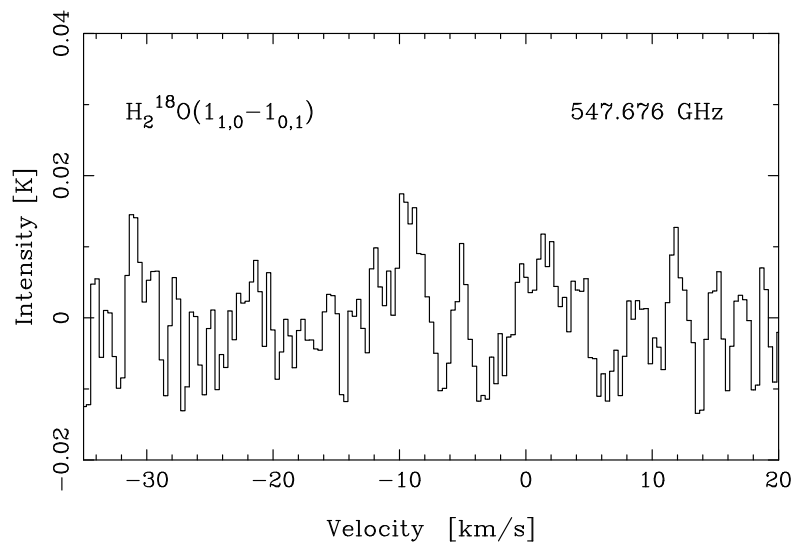

Fig. B.1. Odin observations of $\mathrm{H}_{2}^{18} \mathrm{O}$ in the central position.

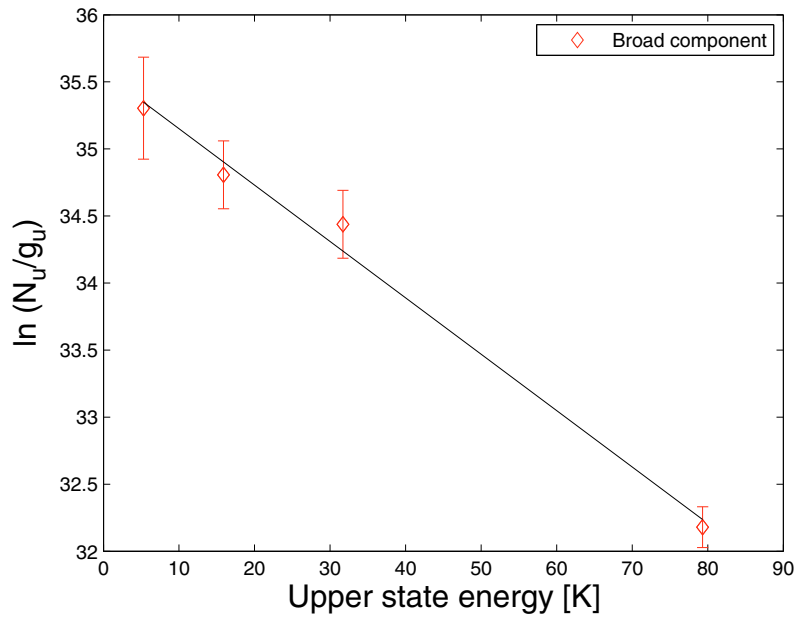

Fig. B.2. Rotation diagram of the broad component of ${ }^{13} \mathrm{CO}(1-0)$ with the Onsala 20-m telescope, $J=2-1$ and $J=3-2$ from Minchin et al. (1993), and $J=5-4$ with Odin, producing $T_{\mathrm{ROT}}=24 \pm 2 \mathrm{~K}$ and $N_{\mathrm{ROT}}=$ $(2.5 \pm 0.4) \times 10^{16} \mathrm{~cm}^{-2}$.

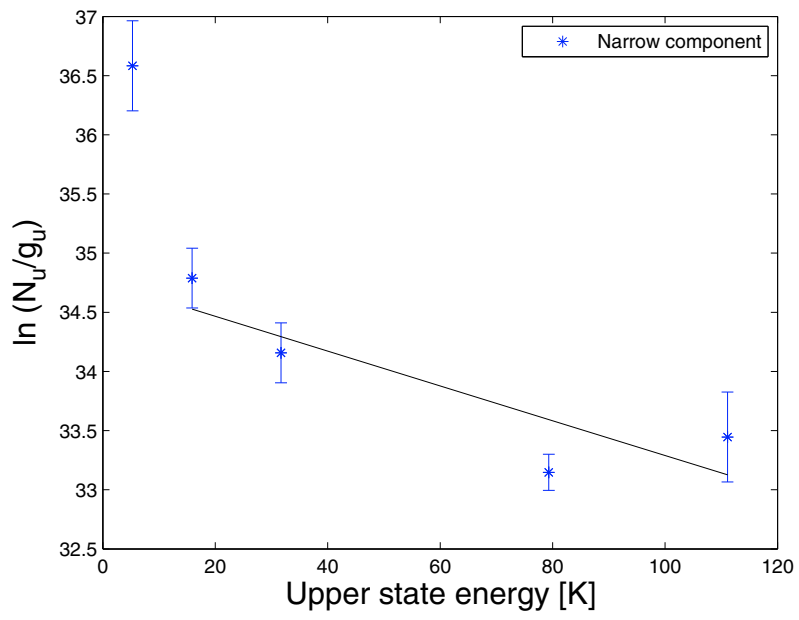

Fig. B.3. Rotation diagram of the narrow component of ${ }^{13} \mathrm{CO}(2-1)$ and $J=3-2$ from Minchin et al. (1993), $J=5-4$ with Odin, and $J=6-5$ from Graf et al. (1993), producing $T_{\mathrm{ROT}}=69 \pm 27 \mathrm{~K}$ and $N_{\mathrm{ROT}}=(3.2 \pm$ 1.8) $\times 10^{16} \mathrm{~cm}^{-2} . J=1-0$ is not included in the fit.

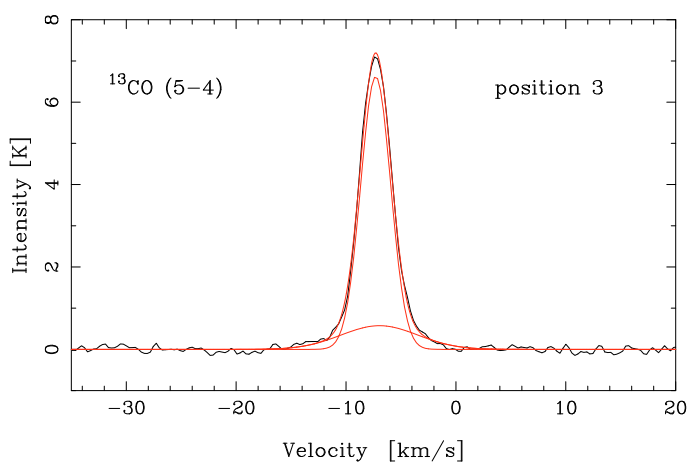

Fig. B.4. Gaussian fits to ${ }^{13} \mathrm{CO}(5-4)$ at the central position. The widths, amplitudes and centre velocities are $3.2 \mathrm{~km} \mathrm{~s}^{-1}$ and $8.2 \mathrm{~km} \mathrm{~s}^{-1} ; 6.610 \mathrm{~K}$ and $0.612 \mathrm{~K} ;-7.3 \mathrm{~km} \mathrm{~s}^{-1}$ and $-6.8 \mathrm{~km} \mathrm{~s}^{-1}$, respectively.

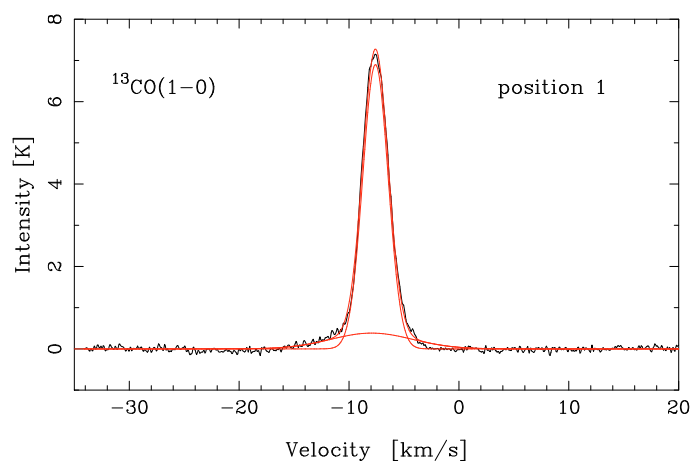

Fig. B.5. Gaussian fits to the convolved (to the Odin 126" beam) spectra of ${ }^{13} \mathrm{CO}(1-0)$ at position 1 . The widths, amplitudes and centre velocities are $2.7 \mathrm{~km} \mathrm{~s}^{-1}$ and $8.6 \mathrm{~km} \mathrm{~s}^{-1} ; 6.982 \mathrm{~K}$ and $0.395 \mathrm{~K} ;-7.6 \mathrm{~km} \mathrm{~s}^{-1}$ and $-8.0 \mathrm{~km} \mathrm{~s}^{-1}$, respectively.

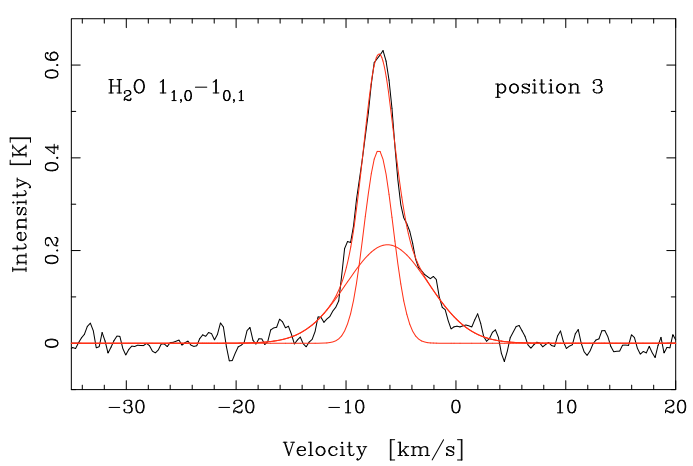

Fig. B.6. Gaussian fits to $\mathrm{H}_{2} \mathrm{O}$ at the central position. The widths, amplitudes and centre velocities are $3.1 \mathrm{~km} \mathrm{~s}^{-1}$ and $8.8 \mathrm{~km} \mathrm{~s}^{-1} ; 416 \mathrm{mK}$ and $213 \mathrm{mK} ;-7.1 \mathrm{~km} \mathrm{~s}^{-1}$ and $-6.1 \mathrm{~km} \mathrm{~s}^{-1}$, respectively.

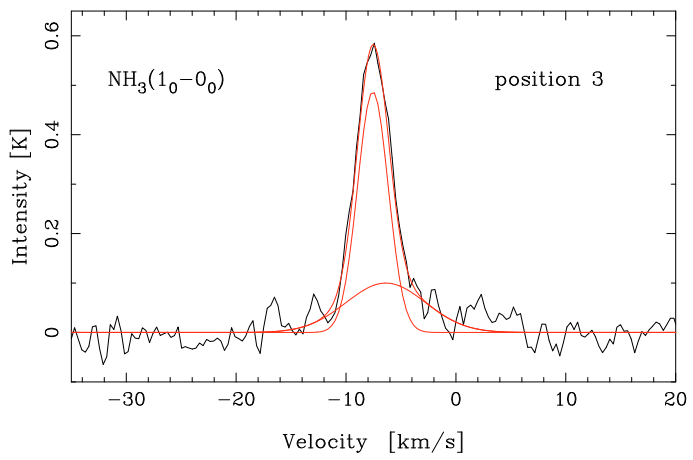

Fig. B.7. Gaussian fits to $\mathrm{NH}_{3}$ at the central position. The widths, amplitudes and centre velocities are $3.3 \mathrm{~km} \mathrm{~s}^{-1}$ and $8.5 \mathrm{~km} \mathrm{~s}^{-1} ; 487 \mathrm{mK}$ and $100 \mathrm{mK} ;-7.6 \mathrm{~km} \mathrm{~s}^{-1}$ and $-6.4 \mathrm{~km} \mathrm{~s}^{-1}$, respectively. 
C. M. Persson et al.: Water and ammonia abundances in S140 with the Odin satellite, Online Material p 3

Table B.1. Observed transitions and their parameters ${ }^{a}$ in S140 with the Odin satellite in a five point NE-SW strip.

\begin{tabular}{|c|c|c|c|c|c|c|c|c|c|c|c|}
\hline Species & Transition & $\begin{array}{l}\text { Freq } \\
{[\mathrm{GHz}]}\end{array}$ & $\begin{array}{l}E_{\mathrm{u}} \\
{[\mathrm{K}]}\end{array}$ & $\begin{array}{l}A \text {-coeff } \\
{\left[\mathrm{s}^{-1}\right]}\end{array}$ & $\begin{array}{l}\Delta v \\
{\left[\mathrm{kms}^{-1}\right]}\end{array}$ & Pos. & $\begin{array}{r}v_{\text {LSR }} \\
{\left[\mathrm{kms}^{-1}\right]}\end{array}$ & $\begin{array}{l}T_{\text {peak }} \\
{[\mathrm{mK}]}\end{array}$ & $\int_{\left[\mathrm{K} \mathrm{kms}^{-1}\right]} T_{\mathrm{A}}^{*} \mathrm{~d} v$ & $\begin{array}{r}\mathrm{rms} \\
{[\mathrm{mK}]}\end{array}$ & note \\
\hline \multirow[t]{5}{*}{$\overline{\mathrm{H}_{2} \mathrm{O}}$} & $1_{1,0}-1_{0,1}$ & 556.936 & 61.0 & $3.46 \mathrm{e}-3$ & 0.27 & 1 & -8.0 & 207 & 0.64 & 39 & \\
\hline & & & & & & 2 & -6.9 & 517 & 2.74 & 68 & \\
\hline & & & & & & 3 & -6.6 & 632 & 3.34 & 19 & \\
\hline & & & & & & 4 & -7.0 & 379 & 1.84 & 57 & \\
\hline & & & & & & 5 & - & - & - & 44 & No detection. \\
\hline $\mathrm{H}_{2}^{18} \mathrm{O}$ & $1_{1,0}-1_{0,1}$ & 547.676 & 60.5 & $3.29 \mathrm{e}-3$ & 0.34 & 3 & - & - & - & $\sim 8$ & No detection. \\
\hline \multirow[t]{5}{*}{$\mathrm{CO}$} & $5-4$ & 576.258 & 83.0 & $1.22 \mathrm{e}-5$ & 0.32 & 1 & -5.4 & 6680 & 47.1 & 67 & \\
\hline & & & & & & 2 & -5.0 & 11760 & 94.2 & 47 & \\
\hline & & & & & & 3 & -5.5 & 13240 & 108.8 & 43 & \\
\hline & & & & & & 4 & -6.6 & 8690 & 60.1 & 50 & \\
\hline & & & & & & 5 & -6.9 & 2220 & 10.1 & 160 & \\
\hline \multirow[t]{5}{*}{${ }^{13} \mathrm{CO}$} & $5-4$ & 550.926 & 79.3 & $1.10 \mathrm{e}-5$ & 0.27 & 1 & -7.8 & 2740 & 8.29 & 115 & \\
\hline & & & & & & 2 & -7.4 & 5930 & 21.4 & 81 & \\
\hline & & & & & & 3 & -7.4 & 7110 & 27.7 & 61 & \\
\hline & & & & & & 4 & -7.6 & 3640 & 13.2 & 119 & \\
\hline & & & & & & 5 & -8.5 & 1190 & 2.5 & 291 & \\
\hline \multirow[t]{5}{*}{$\mathrm{NH}_{3}$} & $1_{0}-0_{0}$ & 572.498 & 27.5 & $1.61 \mathrm{e}-3$ & 0.32 & 1 & -8.0 & 444 & 1.18 & 99 & \\
\hline & & & & & & 2 & -7.4 & 467 & 1.83 & 33 & \\
\hline & & & & & & 3 & -7.4 & 588 & 2.66 & 29 & \\
\hline & & & & & & 4 & -7.3 & 381 & 1.86 & 36 & \\
\hline & & & & & & 5 & - & - & - & 91 & No detection \\
\hline
\end{tabular}

${ }^{a}$ Transition = the quantum numbers for the transition; Freq = rest frequency of the transition; $E_{\mathrm{u}}=$ the upper state energy; $A$-coeff $=$ the Einstein $A$-coefficient; $\Delta v=$ the velocity resolution; Pos $=$ the strip position from NE to SW. $v_{\mathrm{LSR}}=$ the peak LSR velocity; $T_{\text {peak }}=$ the observed peak temperature of the transition; $\int T_{\mathrm{A}}^{*} \mathrm{~d} v=$ the integrated intensity from the observed spectra not corrected for beam-efficiency or beam-filling; rms $=$ noise

Table B.2. ${ }^{13} \mathrm{CO}$ Gaussian fits ${ }^{a}$. $T_{\mathrm{b}}$ uses a source size for the PDR (narrow component) of $120^{\prime \prime} \rightarrow \eta_{\mathrm{bf}}=2$, and a source size for the broad outflow component of $85^{\prime \prime} \rightarrow \eta_{\mathrm{bf}}=3$.

\begin{tabular}{rrrrrrrr}
\hline \hline Pos. & $\begin{array}{r}v_{\text {LSR }} \\
{\left[\mathrm{km} \mathrm{s}^{-1}\right]}\end{array}$ & $\begin{array}{r}\text { err } \\
{\left[\mathrm{km} \mathrm{s}^{-1}\right]}\end{array}$ & $\begin{array}{r}T_{\mathrm{A}}^{*} \\
{[\mathrm{mK}]}\end{array}$ & $\begin{array}{r}T_{\mathrm{b}} \\
{[\mathrm{mK}]}\end{array}$ & $\begin{array}{r}\text { err } \\
{[\mathrm{mK}]}\end{array}$ & $\begin{array}{r}\Delta v \\
{\left[\mathrm{~km} \mathrm{~s}^{-1}\right]}\end{array}$ & $\begin{array}{r}\text { err } \\
{\left[\mathrm{km} \mathrm{s}^{-1}\right]}\end{array}$ \\
\hline 1 & -7.7 & 0.04 & 2665 & 5920 & 68 & 2.8 & 0.08 \\
2 & -7.3 & 0.02 & 5877 & 13060 & 56 & 3.3 & 0.04 \\
3 & -7.3 & 0.01 & 6610 & 14690 & 199 & 3.2 & 0.07 \\
3 & -6.8 & 0.33 & 612 & 2040 & 207 & 8.2 & 1.54 \\
4 & -7.3 & 0.03 & 3620 & 8040 & 66 & 3.5 & 0.07 \\
5 & -8.2 & 0.25 & 1124 & 2500 & 282 & 2.1 & 0.62 \\
\hline
\end{tabular}

${ }^{a}$ Gaussian fits (including errors) to the spectra in 5 positions. For most positions two Gaussians are needed to fit the spectra. Parameters: $v_{\mathrm{LSR}}=$ the LSR velocity at the peak temperature, $T_{\mathrm{A}}^{*}=$ the amplitude of the Gaussian fit, $\Delta v=$ FWHM line width.

Table B.3. $\mathrm{H}_{2} \mathrm{O}$ Gaussian fits ${ }^{a}$. $T_{\mathrm{b}}(\mathrm{PDR})$ uses a source size of $120^{\prime \prime} \rightarrow \eta_{\mathrm{bf}}=2$, while $T_{\mathrm{b}}$ (outflow) uses a source size of $85^{\prime \prime} \rightarrow \eta_{\mathrm{bf}}=3$.

\begin{tabular}{rrrrrrrr}
\hline \hline Pos. & $\begin{array}{r}v_{\text {LSR }} \\
{\left[\mathrm{km} \mathrm{s}^{-1}\right]}\end{array}$ & $\begin{array}{r}\text { err } \\
{\left[\mathrm{km} \mathrm{s}^{-1}\right]}\end{array}$ & $\begin{array}{r}T_{\mathrm{A}}^{*} \\
{[\mathrm{mK}]}\end{array}$ & $\begin{array}{r}T_{\mathrm{b}} \\
{[\mathrm{mK}]}\end{array}$ & $\begin{array}{r}\text { err } \\
{[\mathrm{mK}]}\end{array}$ & $\begin{array}{r}\Delta v \\
{\left[\mathrm{~km} \mathrm{~s}^{-1}\right]}\end{array}$ & $\begin{array}{r}\text { err } \\
{\left[\mathrm{km} \mathrm{s}^{-1}\right]}\end{array}$ \\
\hline 1 & -7.5 & 0.10 & 180 & 400 & 12 & 3.1 & 0.2 \\
2 & -6.9 & 0.08 & 340 & 756 & 28 & 3.5 & 0.3 \\
& -5.4 & 0.39 & 171 & 570 & 27 & 8.8 & 0.7 \\
3 & -7.1 & 0.06 & 416 & 924 & 30 & 3.1 & 0.20 \\
& -6.1 & 0.24 & 213 & 710 & 31 & 8.8 & 0.60 \\
4 & -7.1 & 0.08 & 316 & 702 & 44 & 3.5 & 0.4 \\
& -6.6 & 0.8 & 78 & 260 & 45 & 10.0 & 4.1 \\
\hline
\end{tabular}

${ }^{a}$ Notation as in Table B.2.

Table B.4. $\mathrm{NH}_{3}$ Gaussian fits ${ }^{a}$. $T_{\mathrm{b}}(\mathrm{PDR})$ uses a source size of $120^{\prime \prime} \rightarrow \eta_{\mathrm{bf}}=2$, while $T_{\mathrm{b}}$ (outflow) uses a source size of $85^{\prime \prime} \rightarrow \eta_{\mathrm{bf}}=3$.

\begin{tabular}{lrrrrrrr}
\hline \hline Position & $\begin{array}{r}v_{\text {LSR }} \\
{\left[\mathrm{km} \mathrm{s}^{-1}\right]}\end{array}$ & $\begin{array}{r}\text { err } \\
{\left[\mathrm{km} \mathrm{s}^{-1}\right]}\end{array}$ & $\begin{array}{r}T_{\mathrm{A}}^{*} \\
{[\mathrm{mK}]}\end{array}$ & $\begin{array}{r}T_{\mathrm{b}} \\
{[\mathrm{mK}]}\end{array}$ & $\begin{array}{r}\text { err } \\
{[\mathrm{mK}]}\end{array}$ & $\begin{array}{r}\Delta v \\
{\left[\mathrm{~km} \mathrm{~s}^{-1}\right]}\end{array}$ & $\begin{array}{r}\text { err } \\
{\left[\mathrm{km} \mathrm{s}^{-1}\right]}\end{array}$ \\
\hline 1 & -7.5 & 0.14 & 438 & 973 & 46 & 2.6 & 0.3 \\
2 & -7.9 & 0.25 & 412 & 916 & 200 & 3.3 & 0.9 \\
& -6.1 & 3.4 & 111 & 370 & 160 & 5.8 & 2.6 \\
3 & -7.6 & 0.12 & 487 & 1080 & 90 & 3.3 & 0.50 \\
& -6.4 & 1.4 & 100 & 333 & 92 & 8.5 & 4.3 \\
4 & -7.4 & 0.23 & 321 & 713 & 185 & 3.9 & 1.2 \\
& -6.8 & 2.6 & 61 & 203 & 192 & 8.7 & 12.1 \\
\hline
\end{tabular}

${ }^{a}$ Notation as in Table B.2. 